\title{
Lie algebroid structures on a class of affine bundles
}

\author{
W. Sarlet*, T. Mestdag \\ Department of Mathematical Physics and Astronomy \\ Ghent University, Krijgslaan 281, B-9000 Ghent, Belgium \\ E. Martínez \\ Departamento de Matemática Aplicada \\ Universidad de Zaragoza, María de Luna 3, E-50015 Zaragoza, Spain
}

Keywords: Lie algebroids, affine bundles, exterior calculus.

AMS classification: 37J99, 17B60, 53C15

\begin{abstract}
We introduce the notion of a Lie algebroid structure on an affine bundle whose base manifold is fibred over $\mathbb{R}$. It is argued that this is the framework which one needs for coming to a time-dependent generalization of the theory of Lagrangian systems on Lie algebroids. An extensive discussion is given of a way one can think of forms acting on sections of the affine bundle. It is further shown that the affine Lie algebroid structure gives rise to a coboundary operator on such forms. The concept of admissible curves and dynamical systems whose integral curves are admissible, brings an associated affine bundle into the picture, on which one can define in a natural way a prolongation of the original affine Lie algebroid structure.
\end{abstract}

*email: willy.sarlet@rug.ac.be 


\section{Introduction}

There has been a lot of interest recently in the study of dynamical systems which have a Lie algebroid as carrying space (see e.g. [1, 2, 3, 8, 10, 13]). A Lie algebroid is a vector bundle $\pi: V \rightarrow M$, which comes equipped with two operators. To begin with, there is a bracket operation on $\operatorname{Sec}(\pi)$, the set of sections of $\pi$, which provides it with a real Lie algebra structure. Secondly, there is a linear bundle map $\rho: V \rightarrow T M$, called the anchor map, which establishes a Lie algebra homomorphism between $\operatorname{Sec}(\pi)$ and the real Lie algebra of vector fields on $M$ and does this in such a way that there is a certain compatibility also with the module structure over $C^{\infty}(M)$. To be precise, we have

$$
[\rho(\sigma), \rho(\eta)]=\rho([\sigma, \eta]) \quad \text { and } \quad[\sigma, f \eta]=f[\sigma, \eta]+\rho(\sigma)(f) \eta
$$

for all $\sigma, \eta \in \operatorname{Sec}(\pi)$ and $f \in C^{\infty}(M)$.

Weinstein's paper on Lagrangian mechanics and groupoids 13 roused new interest into the field of algebroids and groupoids. Weinstein introduces 'Lagrangian systems' on a Lie algebroid by means of a Legendre-type map from $V$ to $V^{*}$, associated to a given function $L$ on $V$. The local coordinate expression of such equations reads:

$$
\begin{aligned}
\dot{x}^{i} & =\rho_{\alpha}^{i}(x) y^{\alpha} \\
\frac{d}{d t}\left(\frac{\partial L}{\partial y^{\alpha}}\right) & =\rho_{\alpha}^{i} \frac{\partial L}{\partial x^{i}}-C_{\alpha \beta}^{\gamma} y^{\beta} \frac{\partial L}{\partial y^{\gamma}}
\end{aligned}
$$

where the $x^{i}$ are coordinates on $M, y^{\alpha}$ are fibre coordinates on $V$ and the $C_{\alpha \beta}^{\gamma}$ are structure functions coming from the Lie algebroid structure. Applications for such model equations

can be found e.g. in the theory of systems with symmetries on principal fibre bundles and in rigid body dynamics. Note that, more generally, equations of the form

$$
\begin{aligned}
\dot{x}^{i} & =\rho_{\alpha}^{i}(x) y^{\alpha}, \\
\dot{y}^{\alpha} & =f^{\alpha}(x, y),
\end{aligned}
$$

were called "second-order equations on a Lie algebroid" by Weinstein. They are indeed, to some extent, the analogues of second-order dynamics on a tangent bundle. It is clear, however, that these equations truly are second-order differential equations only when the base manifold and the fibres have the same dimension and $\rho$ is injective. We will therefore rather call them 'pseudo-second-order ordinary differential equations', pseudo-SoDEs for short. Weinstein also raised the question whether there would be a geometrical way of defining equations of the form (1), much in the line of the geometrical construction of classical Lagrange equations, which makes use of the intrinsic structures living on a tangent bundle.

One of us has recently resolved this issue [10] by introducing a kind of lifted Lie algebroid, where suitable analogues can be introduced of the dilation vector field and the vertical endomorphism on a tangent bundle.

In the present paper, we wish to set the stage for an appropriate generalization of this theory to non-autonomous systems of differential equations. We believe that, for example at the level of pseudo-second-order equations, the right generalization is not just a 
matter of allowing the functions $\rho_{\alpha}^{i}$ and $f^{\alpha}$ to depend on time, but rather should produce equations of the form:

$$
\begin{aligned}
\dot{x}^{i} & =\rho_{\alpha}^{i}(t, x) y^{\alpha}+\lambda^{i}(t, x) \\
\dot{y}^{\alpha} & =f^{\alpha}(t, x, y)
\end{aligned}
$$

The reason for this simply is that we wish the structure of the equations to be invariant under time-dependent coordinate transformations. As for Lagrange-type equations, our only concern at the moment is to have an idea of what a time-dependent generalization of (1) should look like. Now, there is a way of developing a kind of formal calculus of variations approach which leads to equations of the form (1), and in which the first set of equations are treated as constraints. We have shown in [12 that if such an approach is adopted when the Lagrangian is allowed to depend on time and the constraints are as in (3), one obtains equations of the form

$$
\begin{aligned}
\dot{x}^{i} & =\rho_{\alpha}^{i}(t, x) y^{\alpha}+\lambda^{i}(t, x) \\
\frac{d}{d t}\left(\frac{\partial L}{\partial y^{\alpha}}\right) & =\rho_{\alpha}^{i} \frac{\partial L}{\partial x^{i}}-\left(C_{\alpha \beta}^{\gamma} y^{\beta}-C_{\alpha}^{\gamma}\right) \frac{\partial L}{\partial y^{\gamma}}
\end{aligned}
$$

where the functions $\rho_{\alpha}^{i}, \lambda^{i}, C_{\alpha \beta}^{\gamma}, C_{\alpha}^{\gamma}$ satisfy the relations

$$
\begin{aligned}
\rho_{\alpha}^{i} \frac{\partial \rho_{\beta}^{j}}{\partial x^{i}}-\rho_{\beta}^{i} \frac{\partial \rho_{\alpha}^{j}}{\partial x^{i}} & =\rho_{\gamma}^{j} C_{\alpha \beta}^{\gamma}, \\
\frac{\partial \rho_{\beta}^{j}}{\partial t}+\lambda^{i} \frac{\partial \rho_{\beta}^{j}}{\partial x^{i}}-\rho_{\beta}^{i} \frac{\partial \lambda^{j}}{\partial x^{i}} & =\rho_{\alpha}^{j} C_{\beta}^{\alpha} .
\end{aligned}
$$

Thus, we want to address the question of explaining the nature of the conditions (5, 6), which presumably should again have something to do with a Lie algebroid structure.

Inspired by these analytical considerations, we will introduce the notion of a Lie algebroid structure on an affine bundle $\pi: E \rightarrow M$, where the base manifold $M$ in addition is assumed to be fibred over $\mathbb{R}$. For the present paper, we will limit ourselves to a number of basic features of such a theory. In particular, we shall show in Section 3 that our defining relations for an affine Lie algebroid are fully consistent with the expectation of being able to develop an exterior differential calculus of sections of the extended dual of this bundle (and its exterior products). We shall further show in Section 4 that vector fields on $E$, whose integral curves are 'admissible curves' and which in fact model differential equations of the pseudo-SoDE type, can be identified in a natural way with special sections of a kind of prolongation of the original affine bundle. This then brings us to a final test, for this paper, of the internal coherence of the newly defined structures: we will verify in Section 5 whether the prolongation of a Lie algebroid, as constructed in [10 for the vector bundle situation, carries over to the present more general situation.

The final section lists a number of other topics of interest, which will be the subject of forthcoming publications. One of our objectives is to arrive at an intrinsic geometrical construction of the time-dependent Lagrangian equations of type (4). For the time being, however, the conditions (5,6) merely serve as benchmarks, to be met by our model of an affine Lie algebroid. 
The basic ingredients for our theory are an affine bundle $\pi: E \rightarrow M$, where the base manifold $M$ is further fibred over $\mathbb{R}$. In Section 2, we define the concept of a Lie algebroid on $\pi$. In Section 3, we show that the axioms for such a Lie algebroid structure give rise to a consistent development of an exterior calculus on sections of the extended dual of $E$. In Section 4, we discuss a special class of curves on $E$, which are said to be admissible by the anchor map and we look into the concept of dynamical systems whose integral curves all belong to this special class. In Section 5, we define the prolongation $\pi_{1}: J_{\lambda}^{1} E \rightarrow E$ of $\pi: E \rightarrow M$ and show that it inherits the Lie algebroid structure from $\pi$.

\section{Affine Lie algebroids}

Let $M$ be an $(n+1)$-dimensional smooth manifold, which is fibred over $\mathbb{R}, \tau: M \rightarrow \mathbb{R}$. We denote the first jet bundle of $\tau$ by $\tau_{1}^{0}: J^{1} M \rightarrow M$. It is an affine bundle modelled on the bundle of tangent vectors to $M$ which are vertical with respect to $\tau$; this vector bundle will be denoted by $\bar{\tau}_{1}^{0}: V M \rightarrow M$. To fix notations further, if $\pi: E \rightarrow M$ is an affine bundle and $\bar{\pi}: V \rightarrow M$ its associated vector bundle, sections of $\pi$ will be denoted by ordinary Greek characters, whereas boldface Greek type will be used for sections of $\bar{\pi}$.

Definition 1. An affine Lie algebroid over $M$ is an affine bundle $\pi: E \rightarrow M$, with the following properties:

1. $\operatorname{Sec}(\bar{\pi})$, the set of sections of the vector bundle $\bar{\pi}: V \rightarrow M$ on which $E$ is modelled, is equipped with a skew-symmetric and bilinear (over $\mathbb{R}$ ) bracket $[\cdot, \cdot]$;

2. the affine space $\operatorname{Sec}(\pi)$ acts by derivations on the real algebra $\operatorname{Sec}(\bar{\pi})$, that is to say, if the same bracket notation $[\zeta, \boldsymbol{\sigma}]$ is used to denote the way $\zeta \in \operatorname{Sec}(\pi)$ acts on $\boldsymbol{\sigma} \in \operatorname{Sec}(\bar{\pi})$, we have $[\zeta, \boldsymbol{\sigma}] \in \operatorname{Sec}(\bar{\pi})$ and

$$
\begin{gathered}
{\left[\zeta, \boldsymbol{\sigma}_{1}+\boldsymbol{\sigma}_{2}\right]=\left[\zeta, \boldsymbol{\sigma}_{1}\right]+\left[\zeta, \boldsymbol{\sigma}_{2}\right], \quad[\zeta+\boldsymbol{\sigma}, \boldsymbol{\eta}]=[\zeta, \boldsymbol{\eta}]+[\boldsymbol{\sigma}, \boldsymbol{\eta}],} \\
{[\zeta,[\boldsymbol{\sigma}, \boldsymbol{\eta}]]=[[\zeta, \boldsymbol{\sigma}], \boldsymbol{\eta}]+[\boldsymbol{\sigma},[\zeta, \boldsymbol{\eta}]] ;}
\end{gathered}
$$

3. there exists an affine bundle map $\lambda: E \rightarrow J^{1} M$ (over the identity on $M$ ), with corresponding vector bundle homomorphism $\rho: V \rightarrow V M$, such that the following compatibility condition holds for all $f \in C^{\infty}(M)$,

$$
[\zeta, f \boldsymbol{\sigma}]=f[\zeta, \boldsymbol{\sigma}]+\lambda(\zeta)(f) \boldsymbol{\sigma} .
$$

Both the affine map $\lambda: E \rightarrow J^{1} M$ and its linear part $\rho: V \rightarrow V M$ will be called anchor maps.

Note that we make no notational distinction between, on the one hand, the affine and linear anchor maps, regarded as maps between total spaces of bundles, and their action on sections of bundles on the other hand. Needless to say, for the interpretation of the 
bracket in the left-hand side of $(12)$, both $\lambda(\zeta)$ and $\rho(\boldsymbol{\sigma})$ are regarded as vector fields on $M$.

Let us derive some further properties which follow from this definition. First of all, if we replace $\zeta$ in (8) by $\zeta+\boldsymbol{\xi}$, for an arbitrary $\boldsymbol{\xi} \in \operatorname{Sec}(\bar{\pi})$, it follows that $\forall \boldsymbol{\xi}, \boldsymbol{\sigma}, \boldsymbol{\eta} \in \operatorname{Sec}(\bar{\pi})$ :

$$
[\boldsymbol{\xi},[\boldsymbol{\sigma}, \boldsymbol{\eta}]]=[[\boldsymbol{\xi}, \boldsymbol{\sigma}], \boldsymbol{\eta}]+[\boldsymbol{\sigma},[\boldsymbol{\xi}, \boldsymbol{\eta}]]
$$

This means, in view of the first hypothesis, that the bracket on $\operatorname{Sec}(\bar{\pi})$ actually provides $\operatorname{Sec}(\bar{\pi})$ with a real Lie algebra structure. Secondly, making the same substitution for $\zeta$ in (9), recalling that $\lambda(\zeta+\boldsymbol{\xi})=\lambda(\zeta)+\rho(\boldsymbol{\xi})$, it follows that

$$
[\boldsymbol{\xi}, f \boldsymbol{\sigma}]=f[\boldsymbol{\xi}, \boldsymbol{\sigma}]+\rho(\boldsymbol{\xi})(f) \boldsymbol{\sigma} .
$$

This means that the linear anchor map $\rho: V \rightarrow V M$ defines a Lie algebra homomorphism from $\operatorname{Sec}(\bar{\pi})$ into the real Lie algebra of vertical vector fields on $M$, and that we have a classical Lie algebroid structure on the vector bundle $\bar{\pi}: V \rightarrow M$ (although its image cannot reach the whole of $T M$ ). Thirdly, replacing $\boldsymbol{\eta}$ by $f \boldsymbol{\eta}$ in (8) and making use of (9) and (11), one obtains the additional compatibility property

$$
[\lambda(\zeta), \rho(\boldsymbol{\sigma})]=\rho([\zeta, \boldsymbol{\sigma}]),
$$

from which it further follows that

$$
[\rho(\boldsymbol{\xi}), \rho(\boldsymbol{\sigma})]=\rho([\boldsymbol{\xi}, \boldsymbol{\sigma}]) .
$$

Remark: for an alternative and equivalent definition of an affine Lie algebroid, we could impose first the Lie algebra structure (10) of the bracket on $\operatorname{Sec}(\bar{\pi})$, together with the compatibility condition (11) for the anchor map $\rho$, and subsequently require that the properties (77-9) hold true for at least one $\zeta \in \operatorname{Sec}(\pi)$ and for an affine map $\lambda: E \rightarrow J^{1} M$ whose linear part is $\rho$. It then follows that such properties hold for all $\zeta$.

We can now further extend the bracket operation to $\operatorname{Sec}(\pi)$, as follows.

Definition 2. (i) For $\boldsymbol{\sigma} \in \operatorname{Sec}(\bar{\pi})$ and $\zeta \in \operatorname{Sec}(\pi)$, we put $[\boldsymbol{\sigma}, \zeta]=-[\zeta, \boldsymbol{\sigma}]$.

(ii) For every two sections $\zeta_{1}, \zeta_{2} \in \operatorname{Sec}(\pi)$ with $\zeta_{12}=\zeta_{2}-\zeta_{1}$ : $\left[\zeta_{1}, \zeta_{2}\right]=\left[\zeta_{1}, \zeta_{12}\right]$.

Observe that the extended bracket in (ii) is a map from $\operatorname{Sec}(\pi) \times \operatorname{Sec}(\pi)$ to $\operatorname{Sec}(\bar{\pi})$. As we will show below, it has Lie algebra type properties, which could justify talking about an "affine Lie algebra structure", were it not that this term is in use already in the literature, with an entirely different meaning. The extended bracket also has Lie algebroid type properties with respect to the anchor maps $\lambda$ and $\rho$.

Proposition 1. The bracket $[\cdot, \cdot]: \operatorname{Sec}(\pi) \times \operatorname{Sec}(\pi) \rightarrow \operatorname{Sec}(\bar{\pi})$, has the following properties:

$$
\begin{aligned}
& {\left[\zeta_{1}, \zeta_{2}+\boldsymbol{\sigma}\right]=\left[\zeta_{1}, \zeta_{2}\right]+\left[\zeta_{1}, \boldsymbol{\sigma}\right],} \\
& {\left[\zeta_{1}, \zeta_{2}\right]=-\left[\zeta_{2}, \zeta_{1}\right],} \\
& {\left[\left[\zeta_{1}, \zeta_{2}\right], \zeta_{3}\right]+\left[\left[\zeta_{2}, \zeta_{3}\right], \zeta_{1}\right]+\left[\left[\zeta_{3}, \zeta_{1}\right], \zeta_{2}\right]=\mathbf{0},} \\
& \rho\left(\left[\zeta_{1}, \zeta_{2}\right]\right)=\left[\lambda\left(\zeta_{1}\right), \lambda\left(\zeta_{2}\right)\right] .
\end{aligned}
$$


ProOF: The first property follows immediately from the definition and (7). Next, we have $\left[\zeta_{2}, \zeta_{1}\right]=\left[\zeta_{2}, \boldsymbol{\zeta}_{21}\right]=-\left[\zeta_{1}+\boldsymbol{\zeta}_{12}, \boldsymbol{\zeta}_{12}\right]=-\left[\zeta_{1}, \zeta_{2}\right]$. For the Jacobi identity, using a simple summation sign to indicate the cyclic sum over the three sections in each summand, we have

$$
\sum\left[\left[\zeta_{1}, \zeta_{2}\right], \zeta_{3}\right]=\sum\left[\left[\zeta_{1}, \zeta_{2}\right], \zeta_{2}+\zeta_{23}\right]=\sum\left[\left[\zeta_{1}, \zeta_{2}\right], \zeta_{23}\right],
$$

in view of the linearity properties and the skew-symmetry of the bracket. Substituting subsequently $\zeta_{1}+\boldsymbol{\zeta}_{12}$ for $\zeta_{2}$, we obtain

$$
\sum\left[\left[\zeta_{1}, \zeta_{2}\right], \zeta_{3}\right]=\sum\left[\left[\zeta_{1}, \zeta_{12}\right], \zeta_{23}\right]
$$

which is zero in view of (8). Finally, the compatibility property (17) easily follows in the same way from the definition of the extended bracket and (12).

To understand what an affine Lie algebroid structure means in coordinates, let us coordinatize $E$ in the usual way, as follows: $t$ denotes the coordinate on $\mathbb{R} ;\left(x^{i}\right)_{1 \leq i \leq n}$ are fibre coordinates on $M$; we further choose a local section $e_{0}$ of $\pi$ to play the role of zero section and a local basis $\left(\boldsymbol{e}_{\alpha}\right)_{1 \leq \alpha \leq k}$ for $\operatorname{Sec}(\bar{\pi})$. Then, if $e$ is a point in the fibre $E_{m}$ over $m \in M$, it can be written in the form: $e=e_{0}(m)+y^{\alpha} \boldsymbol{e}_{\alpha}(m) ;\left(t, x^{i}, y^{\alpha}\right)$ are coordinates of $e\left(\left(t, x^{i}\right)\right.$ being the coordinates of $m$ ).

We have

$$
\left[\boldsymbol{e}_{\alpha}, \boldsymbol{e}_{\beta}\right]=C_{\alpha \beta}^{\gamma}(t, x) \boldsymbol{e}_{\gamma}, \quad\left[e_{0}, \boldsymbol{e}_{\alpha}\right]=C_{\alpha}^{\beta}(t, x) \boldsymbol{e}_{\beta},
$$

for some structure functions $C_{\alpha \beta}^{\gamma}=-C_{\beta \alpha}^{\gamma}$ and $C_{\alpha}^{\beta}$ on $M$. The affine map $\lambda$ and its linear part $\rho$ are fully determined by

$$
\lambda\left(e_{0}\right)=\frac{\partial}{\partial t}+\lambda^{i}(t, x) \frac{\partial}{\partial x^{i}}, \quad \rho\left(\boldsymbol{e}_{\alpha}\right)=\rho_{\alpha}^{i}(t, x) \frac{\partial}{\partial x^{i}} .
$$

The further characterization of the Lie algebroid structure now has the following coordinate translation. The derivation property (8) and the resulting Jacobi identity (10) mean that we have:

$$
\begin{aligned}
& \frac{\partial C_{\alpha \beta}^{\mu}}{\partial t}+\lambda^{i} \frac{\partial C_{\alpha \beta}^{\mu}}{\partial x^{i}}+C_{\alpha \beta}^{\gamma} C_{\gamma}^{\mu}=C_{\alpha \gamma}^{\mu} C_{\beta}^{\gamma}-C_{\beta \gamma}^{\mu} C_{\alpha}^{\gamma}+\rho_{\alpha}^{i} \frac{\partial C_{\beta}^{\mu}}{\partial x^{i}}-\rho_{\beta}^{i} \frac{\partial C_{\alpha}^{\mu}}{\partial x^{i}}, \\
& \sum_{\alpha, \beta, \gamma}\left(\rho_{\alpha}^{i} \frac{\partial C_{\beta \gamma}^{\mu}}{\partial x^{i}}+C_{\alpha \nu}^{\mu} C_{\beta \gamma}^{\nu}\right)=0,
\end{aligned}
$$

where the summation this time refers to a cyclic sum over $\alpha, \beta, \gamma$ and also the compatibility conditions (9), (11) have been invoked. Finally, the properties (12) and (13), for which it is sufficient to express that $\left[\lambda\left(e_{0}\right), \rho\left(\boldsymbol{e}_{\alpha}\right)\right]=\rho\left(\left[e_{0}, \boldsymbol{e}_{\alpha}\right]\right)$ and $\left[\rho\left(\boldsymbol{e}_{\alpha}\right), \rho\left(\boldsymbol{e}_{\beta}\right)\right]=\rho\left(\left[\boldsymbol{e}_{\alpha}, \boldsymbol{e}_{\beta}\right]\right)$, require that

$$
\begin{aligned}
& \frac{\partial \rho_{\beta}^{j}}{\partial t}+\lambda^{i} \frac{\partial \rho_{\beta}^{j}}{\partial x^{i}}-\rho_{\beta}^{i} \frac{\partial \lambda^{j}}{\partial x^{i}}=C_{\beta}^{\alpha} \rho_{\alpha}^{j}, \\
& \rho_{\alpha}^{i} \frac{\partial \rho_{\beta}^{j}}{\partial x^{i}}-\rho_{\beta}^{i} \frac{\partial \rho_{\alpha}^{j}}{\partial x^{i}}=C_{\alpha \beta}^{\gamma} \rho_{\gamma}^{j} .
\end{aligned}
$$


These are precisely the relations (51) and (6) we encountered in the Introduction, in the context of Lagrangian equations of type (4).

It is of some interest to look at the way the various structure and anchor map functions transform under coordinate transformations. There are two distinct levels in making a change of coordinates on $E$, which we will describe separately. Firstly, we could choose a different (local) zero section $\bar{e}_{0}$ and a different local basis $\overline{\boldsymbol{e}}_{\beta}$ for $\operatorname{Sec}(\bar{\pi})$ : say that $\boldsymbol{e}_{\alpha}=$ $A_{\alpha}^{\beta} \overline{\boldsymbol{e}}_{\beta}$ and $e_{0}=\bar{e}_{0}+B^{\alpha} \overline{\boldsymbol{e}}_{\alpha}$. This amounts to making an affine change of coordinates in the fibres of the form: $\bar{y}^{\alpha}=A_{\beta}^{\alpha}(t, x) y^{\beta}+B^{\alpha}(t, x)$. Putting $\left[\overline{\boldsymbol{e}}_{\alpha}, \overline{\boldsymbol{e}}_{\beta}\right]=\bar{C}_{\alpha \beta}^{\gamma} \overline{\boldsymbol{e}}_{\gamma},\left[\bar{e}_{0}, \overline{\boldsymbol{e}}_{\alpha}\right]=\bar{C}_{\alpha}^{\beta} \overline{\boldsymbol{e}}_{\beta}$, and also $\lambda\left(\bar{e}_{0}\right)=\frac{\partial}{\partial t}+\bar{\lambda}^{i} \frac{\partial}{\partial x^{i}}, \rho\left(\overline{\boldsymbol{e}}_{\alpha}\right)=\bar{\rho}_{\alpha}^{j} \frac{\partial}{\partial x^{j}}$, one can verify that the following transformation rules apply:

$$
\rho_{\alpha}^{i}=A_{\alpha}^{\beta} \bar{\rho}_{\beta}^{i}, \quad \bar{\lambda}^{i}=\lambda^{i}-B^{\alpha} \bar{\rho}_{\alpha}^{i}
$$

and further

$$
\begin{aligned}
C_{\alpha \beta}^{\gamma} A_{\gamma}^{\mu} & =\bar{C}_{\gamma \nu}^{\mu} A_{\alpha}^{\gamma} A_{\beta}^{\nu}+\rho_{\alpha}^{i} \frac{\partial A_{\beta}^{\mu}}{\partial x^{i}}-\rho_{\beta}^{i} \frac{\partial A_{\alpha}^{\mu}}{\partial x^{i}} \\
C_{\beta}^{\gamma} A_{\gamma}^{\alpha} & =\bar{C}_{\mu}^{\alpha} A_{\beta}^{\mu}+\bar{C}_{\gamma \mu}^{\alpha} B^{\gamma} A_{\beta}^{\mu}+\frac{\partial A_{\beta}^{\alpha}}{\partial t}+\lambda^{i} \frac{\partial A_{\beta}^{\alpha}}{\partial x^{i}}-\rho_{\beta}^{i} \frac{\partial B^{\alpha}}{\partial x^{i}} .
\end{aligned}
$$

At a different level, one can make a change of coordinates on $M$, of the form: $t^{\prime}=t, x^{\prime i}=$ $x^{\prime i}(t, x)$. This has an effect on the anchor map functions of the form:

$$
\rho_{\alpha}^{\prime j}=\rho_{\alpha}^{i} \frac{\partial x^{\prime j}}{\partial x^{i}}, \quad \lambda^{\prime j}=\frac{\partial x^{\prime j}}{\partial t}+\lambda^{i} \frac{\partial x^{\prime j}}{\partial x^{i}}
$$

A general change of adapted coordinates is of course a composition of the two steps described above.

\section{Exterior calculus on an affine Lie algebroid}

We first recall some features of the by now standard theory of Lie algebroids on a vector bundle (see 91). Considering sections of exterior powers of the dual bundle, one gets a notion of forms on sections of the vector bundle, on which an exterior derivative can be defined which involves the Lie algebroid bracket and the anchor map. It then turns out that the Jacobi identity of the Lie algebroid bracket and the compatibility with the bracket of vector fields via the anchor map are exactly the conditions for this exterior derivative to have the co-boundary property $d^{2}=0$ (see also [6, 17, 10]). In our opinion, such a feature in itself gives a strong indication that the generalization from Lie algebra to Lie algebroid is indeed a meaningful step. We shall therefore investigate in this section whether a similar support can be detected for our extension to Lie algebroids on affine bundles.

The extended dual of the affine space $E_{m}$ is the space of real valued affine functions on $E_{m}$ and will be denoted by $E_{m}^{\dagger}$. The union of these spaces over all points $m \in M$ gives 
us a bundle $\pi^{\dagger}: E^{\dagger} \rightarrow M$ say. Although this is in fact a vector bundle, we are interested in the action of its sections (and sections of its exterior powers) on sections of the affine bundle $\pi$. This brings some subtleties into the picture which need to be investigated in sufficient detail. We will write $\bar{\pi}^{*}: V^{*} \rightarrow M$ for the dual bundle of $\bar{\pi}$ and also use boldface type for its sections (and the sections of its exterior powers). Now, to begin with, if $\theta$ is a section of $\pi^{\dagger}$ and $\zeta \in \operatorname{Sec}(\pi), \theta(\zeta)$ is a function on $M$ defined by $\theta(\zeta)(m)=\theta_{m}\left(\zeta_{m}\right)$. $\theta_{m}$ being an affine function on $E_{m}$, there exists an associated element $\boldsymbol{\theta}_{m} \in V_{m}^{*}$ such that $\forall e_{m} \in E_{m}, \boldsymbol{\sigma}_{m} \in V_{m}$, we have $\theta_{m}\left(e_{m}+\boldsymbol{\sigma}_{m}\right)=\theta_{m}\left(e_{m}\right)+\boldsymbol{\theta}_{m}\left(\boldsymbol{\sigma}_{m}\right)$. Expressed in slightly different terms and now at the level of sections again, a $\theta \in \operatorname{Sec}\left(\pi^{\dagger}\right)$ is such that there exist a $\theta_{0} \in \operatorname{Sec}\left(\pi^{\dagger}\right)$ and a $\boldsymbol{\theta} \in \operatorname{Sec}\left(\bar{\pi}^{*}\right)$, such that for all $\zeta \in \operatorname{Sec}(\pi)$ :

$$
\theta(\zeta)=\theta_{0}\left(\zeta_{0}\right)+\boldsymbol{\theta}(\boldsymbol{\zeta})
$$

where $\zeta_{0}$ is any section and then $\zeta=\zeta_{0}+\zeta$. The two composing elements $\theta_{0}$ (which in fact is simply $\theta$ itself here) and $\boldsymbol{\theta}$ do not depend on the choice of $\zeta_{0}$. With sections of $\pi^{\dagger}$ as our notion of 1 -forms on $\operatorname{Sec}(\pi)$, there is of course no linearity with respect to multiplication by functions on $M$. We can now come in a similar way to the following concept of $k$-forms on $\operatorname{Sec}(\pi)$ (thereby taking for granted that the meaning of a $k$-form on a vector bundle such as $\operatorname{Sec}(\bar{\pi})$ is known).

Definition 3. A $k$-form on the affine bundle $\operatorname{Sec}(\pi)(k \geq 1)$ is a map $\omega: \operatorname{Sec}(\pi) \times$ $\cdots \times \operatorname{Sec}(\pi) \rightarrow C^{\infty}(M)$, for which there exists a $k$-form $\boldsymbol{\omega}$ on the associated vector bundle $\operatorname{Sec}(\bar{\pi})$ and a map $\omega_{0}: \operatorname{Sec}(\pi) \times \operatorname{Sec}(\bar{\pi}) \times \cdots \times \operatorname{Sec}(\bar{\pi}) \rightarrow C^{\infty}(M)$ with the following properties:

1. $\omega_{0}$ is skew-symmetric and $C^{\infty}(M)$-linear in its $k-1$ vector arguments;

2. $\forall \zeta \in \operatorname{Sec}(\pi)$ and $\forall \boldsymbol{\sigma}, \boldsymbol{\zeta}_{j} \in \operatorname{Sec}(\bar{\pi})$, we have

$$
\omega_{0}\left(\zeta+\boldsymbol{\sigma}, \boldsymbol{\zeta}_{1}, \ldots, \boldsymbol{\zeta}_{k-1}\right)=\omega_{0}\left(\zeta, \boldsymbol{\zeta}_{1}, \ldots, \boldsymbol{\zeta}_{k-1}\right)+\boldsymbol{\omega}\left(\boldsymbol{\sigma}, \boldsymbol{\zeta}_{1}, \ldots, \boldsymbol{\zeta}_{k-1}\right)
$$

3. $\forall \zeta_{i} \in \operatorname{Sec}(\pi)$, if we choose an arbitrary $\zeta_{0} \in \operatorname{Sec}(\pi)$ and put $\zeta_{i}=\zeta_{0}+\boldsymbol{\zeta}_{i}$, we have

$$
\omega\left(\zeta_{1}, \ldots, \zeta_{k}\right)=\sum_{i=1}^{k}(-1)^{i-1} \omega_{0}\left(\zeta_{0}, \boldsymbol{\zeta}_{1}, \ldots, \hat{\boldsymbol{\zeta}}_{i}, \ldots, \boldsymbol{\zeta}_{k}\right)+\boldsymbol{\omega}\left(\boldsymbol{\zeta}_{1}, \ldots, \boldsymbol{\zeta}_{k}\right)
$$

There are a number of properties to be checked to make sure that this definition makes sense. First of all, one can verify that with two different choices of a reference section, $\zeta_{0}$ and $\zeta_{0}^{\prime}$ for example, related through $\zeta_{0}=\zeta_{0}^{\prime}+\boldsymbol{\sigma}$, it follows from the second requirement that

$$
\begin{aligned}
& \sum_{i=1}^{k}(-1)^{i-1} \omega_{0}\left(\zeta_{0}, \boldsymbol{\zeta}_{1}, \ldots, \hat{\boldsymbol{\zeta}}_{i}, \ldots, \boldsymbol{\zeta}_{k}\right)+\boldsymbol{\omega}\left(\boldsymbol{\zeta}_{1}, \ldots, \boldsymbol{\zeta}_{k}\right) \\
& \quad=\sum_{i=1}^{k}(-1)^{i-1} \omega_{0}\left(\zeta_{0}^{\prime}, \boldsymbol{\zeta}_{1}^{\prime}, \ldots, \hat{\boldsymbol{\zeta}}_{i}^{\prime}, \ldots, \boldsymbol{\zeta}_{k}^{\prime}\right)+\boldsymbol{\omega}\left(\boldsymbol{\zeta}_{1}^{\prime}, \ldots, \boldsymbol{\zeta}_{k}^{\prime}\right)
\end{aligned}
$$


Secondly, the two elements $\omega_{0}$ and $\boldsymbol{\omega}$ which make up $\omega$ are unique. Indeed, assuming there would be a second couple $\omega_{0}^{\prime}$ and $\boldsymbol{\omega}^{\prime}$ making up the same $\omega$, it follows by choosing $\zeta_{0}=\zeta_{1}$ (such that $\left.\boldsymbol{\zeta}_{1}=0\right)$ that $\omega_{0}=\omega_{0}^{\prime}$, after which it is clear that also $\boldsymbol{\omega}=\boldsymbol{\omega}^{\prime}$. Note finally that the definition implies that $\omega$ itself is skew-symmetric in all its arguments.

The set of forms on $\operatorname{Sec}(\pi)$, which we will denote by $\bigwedge\left(\pi^{\dagger}\right)$, is a module over the ring $C^{\infty}(M)$, which also constitutes the set of 0 -forms. The wedge product of two forms is defined in the usual way. By way of example, if $\alpha$ and $\beta$ are 1-forms, we have

$$
(\alpha \wedge \beta)\left(\zeta_{1}, \zeta_{2}\right)=\alpha\left(\zeta_{1}\right) \beta\left(\zeta_{2}\right)-\alpha\left(\zeta_{2}\right) \beta\left(\zeta_{1}\right),
$$

which for every choice of a reference section $\zeta_{0}$ gives rise to:

$$
\begin{aligned}
(\alpha \wedge \beta)\left(\zeta_{1}, \zeta_{2}\right)= & \alpha\left(\zeta_{0}\right) \boldsymbol{\beta}\left(\boldsymbol{\zeta}_{2}-\boldsymbol{\zeta}_{1}\right)-\beta\left(\zeta_{0}\right) \boldsymbol{\alpha}\left(\boldsymbol{\zeta}_{2}-\boldsymbol{\zeta}_{1}\right) \\
& +(\boldsymbol{\alpha} \wedge \boldsymbol{\beta})\left(\boldsymbol{\zeta}_{1}, \boldsymbol{\zeta}_{2}\right) .
\end{aligned}
$$

It follows that $\boldsymbol{\alpha} \wedge \boldsymbol{\beta}$ is the 2 -form on $\operatorname{Sec}(\bar{\pi})$ corresponding to $\alpha \wedge \beta$, and

$$
(\alpha \wedge \beta)_{0}(\zeta, \boldsymbol{\sigma})=\alpha(\zeta) \boldsymbol{\beta}(\boldsymbol{\sigma})-\beta(\zeta) \boldsymbol{\alpha}(\boldsymbol{\sigma})
$$

Similarly, for the wedge product of three 1-forms, we have

$$
(\alpha \wedge \beta \wedge \gamma)_{0}\left(\zeta, \boldsymbol{\zeta}_{1}, \boldsymbol{\zeta}_{2}\right)=(\alpha(\zeta)(\boldsymbol{\beta} \wedge \gamma)+\beta(\zeta)(\boldsymbol{\gamma} \wedge \boldsymbol{\alpha})+\gamma(\zeta)(\boldsymbol{\alpha} \wedge \boldsymbol{\beta}))\left(\boldsymbol{\zeta}_{1}, \boldsymbol{\zeta}_{2}\right)
$$

These examples suggest to formalize the representation of $k$-forms a bit further. As a preliminary remark, it may sometimes be of interest to extend the interpretation of the operator $\omega_{0}$ in such a way that its single affine section argument need not necessarily be the first. This can simply be achieved by declaring $\omega_{0}$ to be skew-symmetric in all its arguments (but still $C^{\infty}(M)$-linear in its vector arguments only). More importantly, we shall take the sum of $\omega_{0}$-terms in (26) to define another operator, denoted by $\omega^{0}$, as follows:

$$
\omega^{0}\left(\zeta_{1}, \ldots, \zeta_{k}\right)=\sum_{i=1}^{k}(-1)^{i-1} \omega_{0}\left(\zeta_{0}, \boldsymbol{\zeta}_{1}, \ldots, \hat{\boldsymbol{\zeta}}_{i}, \ldots, \boldsymbol{\zeta}_{k}\right)=\sum_{i=1}^{k} \omega_{0}\left(\boldsymbol{\zeta}_{1}, \ldots, \check{\zeta}_{0}, \ldots, \boldsymbol{\zeta}_{k}\right),
$$

where the second expression takes the above remark into account and the symbol $\check{\zeta}_{0}$ then indicates that $\zeta_{0}$ has been inserted in the $i$-th argument. The other important new convention we will adopt is to regard $\boldsymbol{\omega}$ also as acting on affine sections:

$$
\boldsymbol{\omega}\left(\zeta_{1}, \ldots, \zeta_{k}\right)=\boldsymbol{\omega}\left(\zeta_{1}, \ldots, \zeta_{k}\right)
$$

This way, we can formally write

$$
\omega=\omega^{0}+\omega
$$

whereby it is to be understood that the two composing terms $\omega^{0}$ and $\boldsymbol{\omega}$ are not $k$-forms on $\operatorname{Sec}(\pi)$ by themselves. In fact, to compute their value when acting on $k$ sections $\zeta_{i}$, a 
reference section $\zeta_{0}$ has to be chosen, but as argued above, the value of the sum $\omega^{0}+\boldsymbol{\omega}$ in the end does not depend on that choice.

The rather formal looking decomposition (32) now greatly facilitates the representation of wedge products and will make the general coordinate representation of a form more transparant. For example, the result (29) means that

$$
(\alpha \wedge \beta \wedge \gamma)_{0}=\alpha^{0} \otimes(\boldsymbol{\beta} \wedge \gamma)+\beta^{0} \otimes(\boldsymbol{\gamma} \wedge \boldsymbol{\alpha})+\gamma^{0} \otimes(\boldsymbol{\alpha} \wedge \boldsymbol{\beta}),
$$

which then implies from (30) that

$$
(\alpha \wedge \beta \wedge \gamma)^{0}=\alpha^{0} \wedge \boldsymbol{\beta} \wedge \boldsymbol{\gamma}+\boldsymbol{\alpha} \wedge \beta^{0} \wedge \gamma+\boldsymbol{\alpha} \wedge \boldsymbol{\beta} \wedge \gamma^{0}
$$

as expected. More generally, it follows directly from the defining formula for wedge products that for $\omega=\omega^{0}+\boldsymbol{\omega}$ and $\rho=\rho^{0}+\boldsymbol{\rho}$ :

$$
\omega \wedge \rho=\omega^{0} \wedge \rho^{0}+\omega^{0} \wedge \boldsymbol{\rho}+\boldsymbol{\omega} \wedge \rho^{0}+\boldsymbol{\omega} \wedge \boldsymbol{\rho}
$$

where the sum of the first three terms is $(\omega \wedge \rho)^{0}$.

Suppose that, for a coordinatization of $E$, we have chosen a zero section $e_{0}$ and a local basis of vector sections $\boldsymbol{e}_{\alpha}$. Denote by $\left\{\boldsymbol{e}^{\beta}\right\}$ the dual basis for $\operatorname{Sec}\left(\bar{\pi}^{*}\right)$. There exists a global section of $\pi^{\dagger}$ which for each $m$ selects in $E_{m}^{\dagger}$ the constant function 1 . We will call it $e^{0}$. The local zero section $e_{0}$ of $\operatorname{Sec}(\pi)$ can now play the role of the reference section $\zeta_{0}$ in our general considerations. Writing $\zeta=e_{0}+\zeta^{\alpha} \boldsymbol{e}_{\alpha}$ for an arbitrary section $\zeta$, we have for each 1-form $\theta$ : $\theta(\zeta)=\theta\left(e_{0}\right)+\zeta^{\alpha} \boldsymbol{\theta}\left(\boldsymbol{e}_{\alpha}\right)$. Putting $\boldsymbol{\theta}\left(\boldsymbol{e}_{\alpha}\right)=\theta_{\alpha}$ and $\theta\left(e_{0}\right)=\theta_{0}$, we see that $\theta$ has the local representation

$$
\theta=\theta_{0} e^{0}+\theta_{\alpha} e^{\alpha}
$$

where, in agreement with the general decomposition (32), $e^{\alpha}$ has to be regarded now as acting on $\operatorname{Sec}(\pi)$ and $\theta^{0}=\theta_{0} e^{0}$. To be precise, putting

$$
e_{\alpha}=e_{0}+\boldsymbol{e}_{\alpha}
$$

the action of $\boldsymbol{e}^{\beta}$ on affine sections, which can be given a meaning only after introducing a reference section, is determined by:

$$
\boldsymbol{e}^{\beta}\left(e_{0}\right)=0, \quad \boldsymbol{e}^{\beta}\left(e_{\alpha}\right)=\delta_{\alpha}^{\beta} .
$$

There is another slight abuse of notation in (36) since $\theta_{0}$ could have a double meaning: in (36) it represents a local function on $M$, whereas it also could refer to the operator introduced in (24) and more generally in Definition 3. We will, however, seldom use the notation $\theta_{0}$ in the latter sense when dealing with coordinate calculations, so that the meaning will always be clear from the context.

Let us now see how all these notations fit together when we start wedging 1 -forms. For two 1-forms $\alpha=\alpha^{0}+\boldsymbol{\alpha}$ and $\beta=\beta^{0}+\boldsymbol{\beta}$ we find, for example from (28) and (30), that

$$
(\alpha \wedge \beta)^{0}=\alpha^{0} \wedge \boldsymbol{\beta}-\beta^{0} \wedge \boldsymbol{\alpha}
$$


This is in agreement with the general formula (35) since obviously $\alpha^{0} \wedge \beta^{0}=0$. Expressing $\alpha$ and $\beta$ with respect to the basis $\left(e^{0}, \boldsymbol{e}^{\alpha}\right)$, we find

$$
\alpha \wedge \beta=\left(\alpha_{0} \beta_{\gamma}-\beta_{0} \alpha_{\gamma}\right) e^{0} \wedge \boldsymbol{e}^{\gamma}+\frac{1}{2}\left(\alpha_{\gamma} \beta_{\delta}-\alpha_{\delta} \beta_{\gamma}\right) \boldsymbol{e}^{\gamma} \wedge \boldsymbol{e}^{\delta}
$$

Similarly, for the wedge product of three 1-forms with local representations of the form (36), we obtain

$$
\begin{aligned}
\alpha \wedge \beta \wedge \gamma= & \frac{1}{2}\left(\alpha_{0}\left(\beta_{\mu} \gamma_{\nu}-\beta_{\nu} \gamma_{\mu}\right)+\beta_{0}\left(\gamma_{\mu} \alpha_{\nu}-\gamma_{\nu} \alpha_{\mu}\right)\right. \\
& \left.+\gamma_{0}\left(\alpha_{\mu} \beta_{\nu}-\alpha_{\nu} \beta_{\mu}\right)\right) e^{0} \wedge \boldsymbol{e}^{\mu} \wedge \boldsymbol{e}^{\nu}+\boldsymbol{\alpha} \wedge \boldsymbol{\beta} \wedge \boldsymbol{\gamma}
\end{aligned}
$$

It should now be clear without going into any further detail that a general $k$-form on $\operatorname{Sec}(\pi)$ locally has the following representation,

$$
\omega=\frac{1}{(k-1) !} \omega_{0 \mu_{1} \cdots \mu_{k-1}} e^{0} \wedge \boldsymbol{e}^{\mu_{1}} \wedge \cdots \wedge \boldsymbol{e}^{\mu_{k-1}}+\frac{1}{k !} \omega_{\mu_{1} \cdots \mu_{k}} \boldsymbol{e}^{\mu_{1}} \wedge \cdots \wedge \boldsymbol{e}^{\mu_{k}}
$$

where the coefficients are functions on $M$, which are skew-symmetric in all their indices (including the zero for the first term); we have

$$
\begin{aligned}
\omega_{0 \mu_{1} \cdots \mu_{k-1}} & =\omega\left(e_{0}, e_{\mu_{1}}, \ldots, e_{\mu_{k-1}}\right) \\
\omega_{\mu_{1} \cdots \mu_{k}} & =\omega\left(e_{\mu_{1}}, \ldots, e_{\mu_{k}}\right)-\sum_{i=1}^{k} \omega_{\mu_{1} \cdots{\stackrel{0}{i} \cdots \mu_{k}}_{j}}
\end{aligned}
$$

where $\underset{i}{0}$ again means that the index $\mu_{i}$ has been replaced by 0 .

Before arriving at our main goal, the development of an exterior calculus on forms, we will recall a few generalities about derivations. Derivations on $\bigwedge\left(\pi^{\dagger}\right)$ are defined in the usual way. Following the standard work of Frölicher and Nijenhuis [5], one easily shows that derivations are local operators and that they are completely determined by their action on functions and 1-forms. The commutator of two derivations $D_{i}$, of degree $r_{i}$ say, is again a derivation, of degree $r_{1}+r_{2}$, defined by

$$
\left[D_{1}, D_{2}\right]=D_{1} \circ D_{2}-(-1)^{r_{1} r_{2}} D_{2} \circ D_{1}
$$

Perhaps the simplest type of derivation is contraction with a section.

Definition 4. For $\omega \in \bigwedge^{k}\left(\pi^{\dagger}\right)$ and $\zeta \in \operatorname{Sec}(\pi), i_{\zeta} \omega \in \bigwedge^{k-1}\left(\pi^{\dagger}\right)$ is defined by

$$
i_{\zeta} \omega\left(\zeta_{1}, \ldots, \zeta_{k-1}\right)=\omega\left(\zeta, \zeta_{1}, \ldots \zeta_{k-1}\right)
$$

The proof that this is a derivation of degree -1 is standard and does not depend on the pecularities of our present theory. But perhaps we have to convince ourselves in the first place that $i_{\zeta} \omega$ is indeed a form in the sense of Definition 3. 
Proposition 2. $i_{\zeta} \omega$ is a $(k-1)$-form which, in the sense of the general defining relation (20), is determined by an operator $\left(i_{\zeta} \omega\right)_{0}$ and a $k$-form $\mathbf{i}_{\zeta} \boldsymbol{\omega}$ on $S e c(\bar{\pi})$, defined as follows: for all $\boldsymbol{\zeta}_{i} \in \operatorname{Sec}(\bar{\pi}), \zeta_{0} \in \operatorname{Sec}(\pi)$,

$$
\begin{aligned}
\left(i_{\zeta} \omega\right)_{0}\left(\zeta_{0}, \boldsymbol{\zeta}_{2}, \ldots \boldsymbol{\zeta}_{k-1}\right) & =-\omega_{0}\left(\zeta_{0}, \boldsymbol{\zeta}, \boldsymbol{\zeta}_{2}, \ldots \boldsymbol{\zeta}_{k-1}\right), \quad \text { where } \boldsymbol{\zeta}=\zeta-\zeta_{0} \\
\mathbf{i}_{\boldsymbol{\zeta}} \boldsymbol{\omega}\left(\boldsymbol{\zeta}_{2}, \ldots, \boldsymbol{\zeta}_{k}\right) & =\omega_{0}\left(\zeta, \boldsymbol{\zeta}_{2}, \ldots, \boldsymbol{\zeta}_{k}\right) .
\end{aligned}
$$

We further have the property (with $\boldsymbol{\zeta}_{1}=\boldsymbol{\zeta}$ ):

$$
i_{\zeta} \omega\left(\zeta_{2}, \ldots, \zeta_{k}\right)=\sum_{i=1}^{k}(-1)^{i-1}\left(i_{\zeta} \omega_{0}\right)\left(\boldsymbol{\zeta}_{1}, \ldots, \hat{\boldsymbol{\zeta}}_{i}, \ldots, \boldsymbol{\zeta}_{k}\right) .
$$

Proof: A direct computation, using (46) and (26), gives

$$
\begin{aligned}
i_{\zeta} \omega & \left(\zeta_{2}, \ldots, \zeta_{k}\right)=\omega\left(\zeta, \zeta_{2}, \ldots, \zeta_{k}\right) \\
& =\omega_{0}\left(\zeta_{0}, \boldsymbol{\zeta}_{2}, \ldots, \boldsymbol{\zeta}_{k}\right)+\sum_{j=1}^{k-1}(-1)^{j} \omega_{0}\left(\zeta_{0}, \boldsymbol{\zeta}, \boldsymbol{\zeta}_{2}, \ldots, \hat{\boldsymbol{\zeta}}_{j}, \ldots, \boldsymbol{\zeta}_{k}\right)+\boldsymbol{\omega}\left(\boldsymbol{\zeta}, \boldsymbol{\zeta}_{2}, \ldots, \boldsymbol{\zeta}_{k}\right) \\
& =\sum_{j=1}^{k-1}(-1)^{j} \omega_{0}\left(\zeta_{0}, \boldsymbol{\zeta}, \boldsymbol{\zeta}_{2}, \ldots, \hat{\boldsymbol{\zeta}}_{j}, \ldots, \boldsymbol{\zeta}_{k}\right)+\omega_{0}\left(\zeta, \boldsymbol{\zeta}_{2}, \ldots, \boldsymbol{\zeta}_{k}\right),
\end{aligned}
$$

from which we are led to introduce $\left(i_{\zeta} \omega\right)_{0}$ and $\mathbf{i}_{\zeta} \boldsymbol{\omega}$ as in (47) and (48). It is then straightforward to verify that these two operators are linked by a property of type (25), so the first statement follows. Observe that, with an obvious meaning for contraction of the operator $\omega_{0}$ with $\zeta$, we can write: $\mathbf{i}_{\zeta} \boldsymbol{\omega}=i_{\zeta} \omega_{0}$. The somewhat peculiar feature of the additional property is that $i_{\zeta} \omega$ can be completely computed from $i_{\zeta} \omega_{0}$. To prove this we again start from (26) to write (with $\zeta_{1}=\zeta$ )

$$
i_{\zeta} \omega\left(\zeta_{2}, \ldots, \zeta_{k}\right)=\sum_{i=1}^{k}(-1)^{i-1} \omega_{0}\left(\zeta_{0}, \boldsymbol{\zeta}_{1}, \ldots, \hat{\boldsymbol{\zeta}}_{i}, \ldots, \boldsymbol{\zeta}_{k}\right)+\boldsymbol{\omega}\left(\boldsymbol{\zeta}_{1}, \ldots, \boldsymbol{\zeta}_{k}\right)
$$

This time, we substitute $\zeta_{1}-\boldsymbol{\zeta}_{1}$ for $\zeta_{0}$ and observe that the second part of the sum involving $\omega_{0}$, in view of (25) then precisely cancels the last term.

Before we can arrive now at the definition of an exterior derivative operator, we need to give a meaning also to the value of a $k$-form $\omega$, when say its first argument is taken to be a vector section.

Definition 5. If $\omega$ is a $k$-form on $\operatorname{Sec}(\pi)$, then for $\boldsymbol{\sigma} \in \operatorname{Sec}(\bar{\pi})$ and $\zeta_{i} \in \operatorname{Sec}(\pi)$, we put

$$
\omega\left(\boldsymbol{\sigma}, \zeta_{2}, \ldots, \zeta_{k}\right)=\omega\left(\zeta_{1}+\boldsymbol{\sigma}, \zeta_{2}, \ldots, \zeta_{k}\right)-\omega\left(\zeta_{1}, \zeta_{2}, \ldots, \zeta_{k}\right),
$$

where $\zeta_{1}$ is chosen arbitrarily. 
For this to make sense of course, we need to be sure that the result does not depend on the choice of $\zeta_{1}$. Now, if we evaluate the right-hand side of the defining relation by using (26), we obtain

$$
\omega\left(\boldsymbol{\sigma}, \zeta_{2}, \ldots, \zeta_{k}\right)=\boldsymbol{\omega}\left(\boldsymbol{\sigma}, \boldsymbol{\zeta}_{2}, \ldots, \boldsymbol{\zeta}_{k}\right)+\sum_{i=2}^{k}(-1)^{i-1} \omega_{0}\left(\zeta_{0}, \boldsymbol{\sigma}, \boldsymbol{\zeta}_{2}, \ldots, \hat{\boldsymbol{\zeta}}_{i}, \ldots, \boldsymbol{\zeta}_{k}\right)
$$

The right-hand side of this explicit expression makes no mentioning of $\zeta_{1}$ anymore. It might seem at first sight that we have shifted the problem, because it does depend on the reference section $\zeta_{0}$. However, we have argued before that (26) does not depend on the choice of such a reference section, whence our newly defined concept makes sense.

The explicit formula (51) further shows that $i_{\boldsymbol{\sigma}} \omega$ is well defined as a $(k-1)$-form, in the sense of Definition 3. The first term on the right identifies its associated form on $\operatorname{Sec}(\bar{\pi})$, whereas the second term, upon swapping the first two arguments, reveals that $\left(i_{\boldsymbol{\sigma}} \omega\right)_{0}=i_{\boldsymbol{\sigma}} \omega_{0}$. As for local computations, it follows from the definition (50) that $e^{0}(\boldsymbol{\sigma})=$ 0 , whereas the $\boldsymbol{e}^{\alpha}$ act on $\boldsymbol{\sigma}$ simply as duals of $\operatorname{Sec}(\bar{\pi})$.

Definition 6. The exterior derivative of $\omega$, denoted by $d \omega$ is defined by

$$
\begin{aligned}
d \omega\left(\zeta_{1}, \ldots, \zeta_{k+1}\right)= & \sum_{i=1}^{k+1}(-1)^{i-1} \lambda\left(\zeta_{i}\right)\left(\omega\left(\zeta_{1}, \ldots, \hat{\zeta}_{i}, \ldots, \zeta_{k+1}\right)\right) \\
& +\sum_{1 \leq i<j \leq k+1}(-1)^{i+j} \omega\left(\left[\zeta_{i}, \zeta_{j}\right], \zeta_{1}, \ldots, \hat{\zeta}_{i}, \ldots, \hat{\zeta}_{j}, \ldots, \zeta_{k+1}\right) .
\end{aligned}
$$

Note first that we are making use of definition 5 in the second term on the right, because the bracket of two affine sections is a vector section. It is fairly obvious that $d \omega$ is skewsymmetric in all its arguments. To justify the definition, however, we should be able to identify an operator $(d \omega)_{0}$ and a related $(k+1)$-form $\mathbf{d} \boldsymbol{\omega}$ on $\operatorname{Sec}(\bar{\pi})$, such that $d \omega$ satisfies an expression of type (26). We of course have an exterior derivative at our disposal for the $k$-form $\boldsymbol{\omega}$ on $\operatorname{Sec}(\bar{\pi})$ which we denote by $d$ also. We know that $d \boldsymbol{\omega}$ has a property of type (52) (or can be defined that way), with vector sections replacing affine sections and $\rho$ as anchor map instead of $\lambda$.

Definition 7. For $\omega_{0}: \operatorname{Sec}(\pi) \times \operatorname{Sec}(\bar{\pi}) \times \cdots \times \operatorname{Sec}(\bar{\pi}) \rightarrow C^{\infty}(M)$, we define $d \omega_{0}$, an operator of the same type, but depending on one more vector section, by

$$
\begin{aligned}
& d \omega_{0}\left(\zeta, \boldsymbol{\zeta}_{2}, \ldots, \boldsymbol{\zeta}_{k+1}\right)=\lambda(\zeta)\left(\boldsymbol{\omega}\left(\boldsymbol{\zeta}_{2}, \ldots, \boldsymbol{\zeta}_{k+1}\right)\right) \\
& \quad+\sum_{i=2}^{k+1}(-1)^{i-1} \rho\left(\boldsymbol{\zeta}_{i}\right)\left(\omega_{0}\left(\zeta, \boldsymbol{\zeta}_{2}, \ldots, \hat{\boldsymbol{\zeta}}_{i}, \ldots, \boldsymbol{\zeta}_{k+1}\right)\right) \\
& \quad+\sum_{j=2}^{k+1}(-1)^{j+1} \boldsymbol{\omega}\left(\left[\zeta, \boldsymbol{\zeta}_{j}\right], \boldsymbol{\zeta}_{2}, \ldots, \hat{\boldsymbol{\zeta}}_{j}, \ldots, \boldsymbol{\zeta}_{k+1}\right) \\
& \quad-\sum_{2 \leq i<j \leq k+1}(-1)^{i+j} \omega_{0}\left(\zeta,\left[\boldsymbol{\zeta}_{i}, \boldsymbol{\zeta}_{j}\right], \boldsymbol{\zeta}_{2}, \ldots, \hat{\boldsymbol{\zeta}}_{i}, \ldots, \hat{\boldsymbol{\zeta}}_{j}, \ldots, \boldsymbol{\zeta}_{k+1}\right) .
\end{aligned}
$$


This expression may look rather exotic at first, but it is obtained by formally copying the definition (52) and writing in that process either $\lambda$ or $\rho$, and either $\omega_{0}$ or $\boldsymbol{\omega}$, in such a way that every term in the right-hand side has a proper meaning. There are two important observations to be made here. First of all, the required linearity of $d \omega_{0}$ in its vector arguments relies on the properties (9) and (11) of our Lie algebroid bracket. Secondly, replacing the affine section $\zeta$ in the definition by $\zeta+\boldsymbol{\sigma}$, we find:

$$
d \omega_{0}\left(\zeta+\boldsymbol{\sigma}, \boldsymbol{\zeta}_{2}, \ldots, \boldsymbol{\zeta}_{k+1}\right)=d \omega_{0}\left(\zeta, \boldsymbol{\zeta}_{2}, \ldots, \boldsymbol{\zeta}_{k+1}\right)+d \boldsymbol{\omega}\left(\boldsymbol{\sigma}, \boldsymbol{\zeta}_{2}, \ldots, \boldsymbol{\zeta}_{k+1}\right) .
$$

We thus know what to expect for the decomposition (26) of $d \omega$ and this is confirmed by the following result.

Proposition 3. We have $(d \omega)_{0}=d \omega_{0}$ and $\mathbf{d} \boldsymbol{\omega}=d \boldsymbol{\omega}$.

The proof, which involves a rather technical but straightforward calculation, is given in the appendix.

It is of some interest to work out some simple cases in detail. For a function $f \in C^{\infty}(M)$, $d f$ is defined by

$$
d f(\zeta)=\lambda(\zeta)(f)=\lambda\left(\zeta_{0}\right)(f)+\rho(\zeta)(f),
$$

from which we learn that $(d f)_{0}=d f$ (as expected) and $\mathbf{d f}(\boldsymbol{\sigma})=\rho(\boldsymbol{\sigma})(f)$. If $\theta$ is a 1-form, the defining relation (52) for its exterior derivative reads

$$
d \theta\left(\zeta_{1}, \zeta_{2}\right)=\lambda\left(\zeta_{1}\right)\left(\theta\left(\zeta_{2}\right)\right)-\lambda\left(\zeta_{2}\right)\left(\theta\left(\zeta_{1}\right)\right)-\boldsymbol{\theta}\left(\left[\zeta_{1}, \zeta_{2}\right]\right)
$$

Introducing an arbitrary reference section $\zeta_{0}$, it is easy to verify that this can be rewritten as

$$
d \theta\left(\zeta_{1}, \zeta_{2}\right)=(d \theta)_{0}\left(\zeta_{0}, \boldsymbol{\zeta}_{2}\right)-(d \theta)_{0}\left(\zeta_{0}, \boldsymbol{\zeta}_{1}\right)+\mathbf{d} \boldsymbol{\theta}\left(\boldsymbol{\zeta}_{1}, \boldsymbol{\zeta}_{2}\right),
$$

where $\mathbf{d} \boldsymbol{\theta}=d \boldsymbol{\theta}$ and

$$
(d \theta)_{0}(\zeta, \boldsymbol{\sigma})=\lambda(\zeta)(\boldsymbol{\theta}(\boldsymbol{\sigma}))-\rho(\boldsymbol{\sigma})(\theta(\zeta))-\boldsymbol{\theta}([\zeta, \boldsymbol{\sigma}])
$$

This is in perfect agreement with the results of Proposition 3 and Definition 7.

Concerning derivation properties, it is trivial to verify that for the product of functions: $d(f g)=f d g+g d f$. Also, from (56) applied to $f \theta$ we get:

$$
\begin{aligned}
d(f \theta)\left(\zeta_{1}, \zeta_{2}\right) & =\lambda\left(\zeta_{1}\right)\left(f \theta\left(\zeta_{2}\right)\right)-\lambda\left(\zeta_{2}\right)\left(f \theta\left(\zeta_{1}\right)\right)-f \boldsymbol{\theta}\left(\left[\zeta_{1}, \zeta_{2}\right]\right) \\
& =d f\left(\zeta_{1}\right) \theta\left(\zeta_{2}\right)-d f\left(\zeta_{2}\right) \theta\left(\zeta_{1}\right)+f\left(\lambda\left(\zeta_{1}\right)\left(\theta\left(\zeta_{2}\right)\right)-\lambda\left(\zeta_{2}\right)\left(\theta\left(\zeta_{1}\right)\right)-\boldsymbol{\theta}\left(\left[\zeta_{1}, \zeta_{2}\right]\right)\right)
\end{aligned}
$$

from which we conclude that

$$
d(f \theta)=f d \theta+d f \wedge \theta
$$

Recalling now the general statements about derivations we made before, we can conclude that there exists a unique derivation $\hat{d}$ on $\bigwedge\left(\pi^{\dagger}\right)$, of degree 1 , which coincides with our $d$ 
on functions and 1-forms. If we can show that $\hat{d} \omega=d \omega$ for an arbitrary $\omega \in \bigwedge\left(\pi^{\dagger}\right)$, we will know that the operator $d$ defined by (52) is a derivation. To this end, let us introduce

$$
\hat{d}_{\zeta}=\left[i_{\zeta}, \hat{d}\right]
$$

which, as commutator of two derivations, is itself a derivation of degree 0 on $\bigwedge\left(\pi^{\dagger}\right)$. We extend the action of $\hat{d}_{\zeta}$ to $\operatorname{Sec}(\pi)$ 'by duality'. That is to say, for $\eta \in \operatorname{Sec}(\pi), \hat{d}_{\zeta} \eta$ is defined by requiring that for all $\theta \in \bigwedge^{1}\left(\pi^{\dagger}\right)$ :

$$
\left\langle\hat{d}_{\zeta} \eta, \theta\right\rangle=\hat{d}_{\zeta}(\theta(\eta))-\hat{d}_{\zeta} \theta(\eta)
$$

It is easy to see that $\hat{d}_{\zeta} \eta$ is skew-symmetric in $\zeta$ and $\eta$, so that it makes sense to introduce a bracket notation for it: $[\zeta, \eta]^{\wedge}=\hat{d}_{\zeta} \eta$. We now recall a result proved in [11] which, although stated there in an entirely different context, has a quite universal validity.

Lemma 1. Given a derivation $\hat{d}$ of degree 1 , and introducing $\hat{d}_{\zeta}$ and $[\zeta, \eta]^{\wedge}$ as above, we have for all $\omega \in \bigwedge^{k}\left(\pi^{\dagger}\right)$ :

$$
\begin{aligned}
\hat{d} \omega\left(\zeta_{1}, \ldots, \zeta_{k+1}\right)= & \sum_{i=1}^{k+1}(-1)^{i-1} \hat{d}_{\zeta_{i}}\left(\omega\left(\zeta_{1}, \ldots, \hat{\zeta}_{i}, \ldots, \zeta_{k+1}\right)\right) \\
& +\sum_{1 \leq i<j \leq k+1}(-1)^{i+j} \omega\left(\left[\zeta_{i}, \zeta_{j}\right]^{\wedge}, \zeta_{1}, \ldots, \hat{\zeta}_{i}, \ldots, \hat{\zeta}_{j}, \ldots, \zeta_{k+1}\right) .
\end{aligned}
$$

To show that $\hat{d} \omega=d \omega$ now, it suffices to verify that $\hat{d}_{\zeta} f=\lambda(\zeta)(f)$ on functions, and that the bracket $[,]^{\wedge}$ coincides with the Lie algebroid bracket. We have $\hat{d}_{\zeta} f=i_{\zeta} \hat{d} f=i_{\zeta} d f=$ $\lambda(\zeta)(f)$, and for all $\theta \in \bigwedge^{1}\left(\pi^{\dagger}\right)$

$$
\begin{aligned}
\left\langle[\zeta, \eta]^{\wedge}, \theta\right\rangle & =\left\langle\hat{d}_{\zeta} \eta, \theta\right\rangle=\lambda(\zeta)(\theta(\eta))-\hat{d} \theta(\zeta, \eta)-\hat{d} i_{\zeta} \theta(\eta) \\
& =\lambda(\zeta)(\theta(\eta))-\lambda(\eta)(\theta(\zeta))-d \theta(\zeta, \eta)=\boldsymbol{\theta}([\zeta, \eta]),
\end{aligned}
$$

from which the desired result follows.

We now reach the main question which is about the relationship between $d^{2}$ and the compatibility requirements in the definition of an affine Lie algebroid. To appreciate the meaning of the following lemma, we take a step back and assume now that the bracket $\left[\zeta_{i}, \zeta_{j}\right]$ figuring in the definition (52) of $d$ satisfies the 'Leibniz-type property' (9) with respect to the module structure of $\operatorname{Sec}(\pi)$ (and the resulting property (11)), but no further compatibility or Lie algebra conditions a priori. Remember that the property (9) of the bracket was necessary to make sure that $d \omega$ is a form in the first place.

Lemma 2. For all $\omega \in \bigwedge^{k}\left(\pi^{\dagger}\right)$ and $\zeta_{i} \in \operatorname{Sec}(\pi)$, we have

$$
\begin{aligned}
& d^{2} \omega\left(\zeta_{1}, \ldots, \zeta_{k+2}\right)= \\
& \sum_{1 \leq i<j \leq k+2}(-1)^{i+j}\left(\rho\left(\left[\zeta_{i}, \zeta_{j}\right]\right)-\left[\lambda\left(\zeta_{i}\right), \lambda\left(\zeta_{j}\right)\right]\right)\left(\omega\left(\zeta_{1}, \ldots, \hat{\zeta}_{i}, \ldots, \hat{\zeta}_{j}, \ldots, \zeta_{k+2}\right)\right) \\
& \quad+\sum_{1 \leq i<j<l \leq k+2}(-1)^{i+j+l} \omega\left(\sum_{i, j, l}\left[\zeta_{i},\left[\zeta_{j}, \zeta_{l}\right]\right], \zeta_{1}, ., \hat{\zeta}_{i}, ., \hat{\zeta}_{j}, ., \hat{\zeta}_{l}, ., \zeta_{k+2}\right),
\end{aligned}
$$

(where the smaller summation sign of course refers again to a cyclic sum over the three indices involved). 
In fact this lemma, with suitable adaptations, also has a rather universal validity. For completeness, we include a proof in Appendix A.

Proposition 4. The exterior derivative has the property $d^{2}=0$ if and only if the bracket further satisfies the Jacobi identity (10) (or equivalently (8)).

Proof: If (9) and (16) hold true, we also have (8) and know from previous considerations that (12) then holds as well. The above lemma this way trivially implies $d^{2}=0$. For the converse, we observe that $d^{2} f=0$, for $f \in C^{\infty}(M)$, implies (17), from which it subsequently follows that $d^{2} \theta=0$, with $\theta \in \Lambda^{1}\left(\pi^{\dagger}\right)$, implies (16).

It remains now to list coordinate expressions for the basic exterior derivatives. Let $\left(t, x^{i}\right)$ as before be coordinates on $M$. For their exterior derivatives we obtain the following: for all $\zeta \in \operatorname{Sec}(\pi)$

$$
d t(\zeta)=\lambda(\zeta)(t)=1, \quad d x^{i}(\zeta)=\lambda(\zeta)\left(x^{i}\right)
$$

from which it follows that $\mathbf{d t}=\mathbf{0}$ and $\mathbf{d} \mathbf{x}^{\mathbf{i}}(\boldsymbol{\sigma})=\rho(\boldsymbol{\sigma})\left(x^{i}\right)$ (and of course $(d t)_{0}=$ $\left.d t,\left(d x^{i}\right)_{0}=d x^{i}\right)$. In terms of the general representation (36) of a 1 -form, we thus have:

$$
\begin{aligned}
d t & =e^{0} \\
d x^{i} & =\lambda^{i} e^{0}+\rho_{\alpha}^{i} e^{\alpha} .
\end{aligned}
$$

Obviously, we have $d e^{0}=0$. We further calculate, making use, for example, of (43,44), the general formula (56) and the coordinate expressions (18), that

$$
d \boldsymbol{e}^{\alpha}=-C_{\beta}^{\alpha} e^{0} \wedge \boldsymbol{e}^{\beta}-\frac{1}{2} C_{\beta \gamma}^{\alpha} \boldsymbol{e}^{\beta} \wedge \boldsymbol{e}^{\gamma} .
$$

It is instructive to verify that expressing the properties $d^{2} \boldsymbol{e}^{\alpha}=0$ and $d^{2} x^{i}=0$ is indeed equivalent to the requirements (20,21) and (22,23), respectively.

To complete the picture of basic derivations on $\bigwedge\left(\pi^{\dagger}\right)$, we have a closer look at the analogue of the classical Lie derivative.

Definition 8. For every $\zeta \in \operatorname{Sec}(\pi)$, the derivation $d_{\zeta}$ of degree zero is defined as

$$
d_{\zeta}=\left[i_{\zeta}, d\right]=i_{\zeta} \circ d+d \circ i_{\zeta}
$$

So, since $d_{\zeta}$ is defined as a commutator of derivations, we know that it will itself be a derivation of degree zero:

$$
d_{\zeta}(\omega \wedge \mu)=d_{\zeta} \omega \wedge \mu+\omega \wedge d_{\zeta} \mu
$$

Likewise, we can rely on proofs similar to those in the standard theory to conclude that the following commutator properties will hold true:

$$
\left[d_{\zeta}, i_{\eta}\right]=i_{[\zeta, \eta]}, \quad\left[d_{\zeta}, d\right]=0, \quad\left[d_{\zeta}, d_{\eta}\right]=d_{[\zeta, \eta]}
$$


Note, however, that a Lie-type derivation with respect to a vector section turns up in the last property, and this is indeed well defined also as: $d_{\boldsymbol{\sigma}}=\left[i_{\boldsymbol{\sigma}}, d\right]$. It is further natural to extend the action of $d_{\zeta}$ to $\operatorname{Sec}(\pi)$ by duality, i.e. to require that a property of type (60) holds true. It then follows, as expected, that for $\eta, \zeta \in \operatorname{Sec}(\pi)$,

$$
d_{\zeta} \eta=[\zeta, \eta]
$$

As a result of such an extension, $d_{\zeta}$ has Leibniz-type properties also with respect to the evaluation of forms on the appropriate number of affine (or vector) sections; the following property, which could be verified by a direct computation from the definition of $d_{\zeta}$, thus becomes self-evident:

$$
d_{\eta} \omega\left(\zeta_{1}, \ldots, \zeta_{k}\right)=\lambda(\eta)\left(\omega\left(\zeta_{1}, \ldots, \zeta_{k}\right)\right)+\sum_{j=1}^{k}(-1)^{j} \omega\left(\left[\eta, \zeta_{j}\right], \zeta_{1}, \ldots, \hat{\zeta}_{j}, \ldots, \zeta_{k}\right)
$$

In the interest of doing computations, we list the Lie-type derivatives of functions $f \in$ $C^{\infty}(M)$ and the local basis of 1-forms. For $\zeta=e_{0}+\zeta^{\alpha} \boldsymbol{e}_{\alpha}$,

$$
d_{\zeta} f=\lambda(\zeta)(f), \quad d_{\zeta} e^{0}=0, \quad d_{\zeta} \boldsymbol{e}^{\alpha}=C_{\beta}^{\alpha} \zeta^{\beta} e^{0}-C_{\beta}^{\alpha} \boldsymbol{e}^{\beta}+C_{\beta \gamma}^{\alpha} \zeta^{\gamma} \boldsymbol{e}^{\beta}+d \zeta^{\alpha}
$$

For future developments, it may be of some interest, finally, to list what the two composing parts of $d_{\zeta} \omega$ are, in the sense of the defining relation (26) of forms.

Proposition 5. For $\omega \in \bigwedge^{k}\left(\pi^{\dagger}\right)$, we have

$$
\begin{aligned}
\left(d_{\zeta} \omega\right)_{0}\left(\zeta_{0}, \boldsymbol{\zeta}_{1}, \ldots, \boldsymbol{\zeta}_{k-1}\right)= & \lambda(\zeta)\left(\omega_{0}\left(\zeta_{0}, \boldsymbol{\zeta}_{1}, \ldots, \boldsymbol{\zeta}_{k-1}\right)\right)-\boldsymbol{\omega}\left(\left[\zeta, \zeta_{0}\right], \boldsymbol{\zeta}_{1}, \ldots, \boldsymbol{\zeta}_{k-1}\right) \\
& +\sum_{j=1}^{k-1}(-1)^{j} \omega_{0}\left(\zeta_{0},\left[\zeta, \zeta_{j}\right], \boldsymbol{\zeta}_{1}, \ldots, \hat{\boldsymbol{\zeta}}_{j}, \ldots, \boldsymbol{\zeta}_{k-1}\right), \\
\mathbf{d}_{\boldsymbol{\zeta}} \boldsymbol{\omega}\left(\boldsymbol{\zeta}_{1}, \ldots, \boldsymbol{\zeta}_{k}\right)= & \lambda(\zeta)\left(\boldsymbol{\omega}\left(\boldsymbol{\zeta}_{1}, \ldots, \boldsymbol{\zeta}_{k}\right)\right) \\
& +\sum_{j=1}^{k-1}(-1)^{j} \boldsymbol{\omega}\left(\left[\zeta, \zeta_{j}\right], \boldsymbol{\zeta}_{1}, \ldots, \hat{\boldsymbol{\zeta}}_{j} \ldots, \boldsymbol{\zeta}_{k}\right) .
\end{aligned}
$$

These are exactly the sort of expressions one expects. The proof is a matter of a direct computation, starting from the formula (69) and using the decompositions (26) and (51). It further requires manipulations of double sums of the same nature as those in Appendix A.

\section{$4 \lambda$-admissible curves and dynamics}

As we expressed in the introduction, the model of affine Lie algebroids we are developing should in the first place offer an environment in which one can accomodate the timedependent Lagrange-type equations (田). At present, we wish to look in more detail at the 
geometric nature of the more general dynamical systems, which we call pseudo-secondorder equations, and are those described by differential equations of the form (3). For this purpose in fact, we do not need the full machinery of algebroids: it suffices to assume that $E$ is an affine bundle over $M$ and $\lambda: E \rightarrow J^{1} M$ an affine bundle map over the identity.

Definition 9. A curve $\psi$ in $E$, which is a section of $\tau \circ \pi$, is said to be $\lambda$-admissible, if $\lambda \circ \psi=j^{1}(\pi \circ \psi)$.

One could say that $\psi$ is the $\lambda$-prolongation of a curve in $M$. In coordinates, we have

$$
\psi: t \mapsto\left(t, x^{i}(t), y^{\alpha}(t)\right), \quad \text { with } \quad \dot{x}^{i}(t)=\lambda^{i}(t, x(t))+\rho_{\alpha}^{i}(t, x(t)) y^{\alpha}(t) .
$$

Note in passing that, not unexpectedly, one can characterize $\lambda$-admissibility via a concept of contact forms: putting $\Theta^{i}=\lambda^{*} \theta^{i}$, where the $\theta^{i}$ are the contact forms on $J^{1} M$, we have that $\psi$ is a $\lambda$-admissible curve in $E$ if and only if $\psi^{*} \Theta^{i}=0$.

Pseudo-second-order equation fields on $E$ are vector fields whose integral curves all are $\lambda$-admissible curves. As in the standard theory of SoDEs on a tangent bundle or first jet bundle, however, there is a simple direct characterization of such vector fields.

Definition 10. $\Gamma \in \mathcal{X}(E)$ is a pseudo-second-order equation field if

$$
T \pi \circ \Gamma=i \circ \lambda
$$

where $i$ is the injection of $J^{1} M$ into $T M$.

Clearly, in coordinates, a pseudo-SoDE is of the form

$$
\Gamma=\frac{\partial}{\partial t}+\left(\lambda^{i}(t, x)+\rho_{\alpha}^{i}(t, x) y^{\alpha}\right) \frac{\partial}{\partial x^{i}}+f^{\alpha}(t, x, y) \frac{\partial}{\partial y^{\alpha}}
$$

for some functions $f^{\alpha}$, and it is obvious that all its integral curves will be $\lambda$-admissible. The following diagram visualizes the notions of $\lambda$-admissible curves and pseudo-SoDEs.

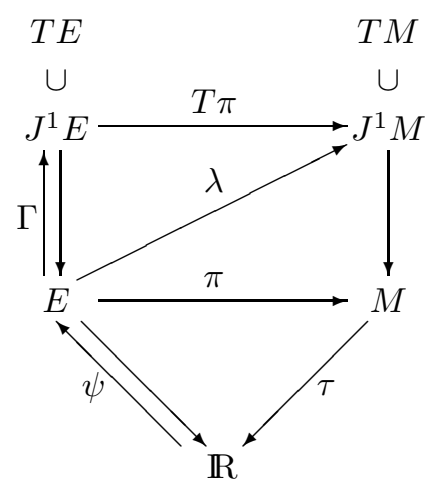

An important point now, however, is that there is a natural way of interpreting the vector field $\Gamma$ as section of a different bundle.

From the above definition, it is clear that a pseudo-SoDE is actually a section of $(\tau \circ \pi)_{1}^{0}$ : $J^{1} E \rightarrow E$, with the additional property that for all $p \in E,\left.T \pi\right|_{J^{1} E}(\Gamma(p))=\lambda(p)$. An 
equivalent way of saying the same thing, by definition of the concept of a pullback bundle, is that $(p, \Gamma(p))$ is a point of $\lambda^{*} J^{1} E$, with $J^{1} E$ regarded as fibred over $J^{1} M$ via $\left.T \pi\right|_{J^{1} E}$. From now on, we will write $J_{\lambda}^{1} E$ for $\lambda^{*} J^{1} E$, and denote its two projections as indicated in the following diagram:

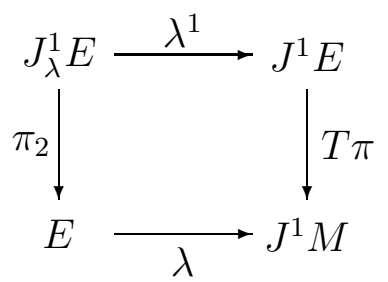

If we finally put $\pi_{1}=(\tau \circ \pi)_{1}^{0} \circ \lambda^{1}$, there is yet another way of expressing the characterization of a pseudo-SoDE. Indeed, from the trivial observation that $(\tau \circ \pi)_{1}^{0}(\Gamma(p))=p=$ $\pi_{2}((p, \Gamma(p)))$, it follows that a pseudo-SodE $\Gamma$ can be regarded also as a section of the bundle $\pi_{1}: J_{\lambda}^{1} E \rightarrow E$, with the property that $\pi_{2} \circ \Gamma=\pi_{1} \circ \Gamma$.

The various spaces and projections, described in this discussion, are depicted in the diagram of the next section. This diagram immediately suggests the following question: if we put more structure into the scheme by assuming now again that $\pi: E \rightarrow M$ carries an affine Lie algebroid structure, is it possible to prolong this structure to the bundle $\pi_{1}: J_{\lambda}^{1} E \rightarrow E$, in such a way of course that $\lambda^{1}$ becomes the anchor map of the induced affine Lie algebroid?

\section{Prolongation of affine Lie algebroids}

We shall now look in more detail at the bundle $\pi_{1}: J_{\lambda}^{1} E \rightarrow E$. Its total space is the manifold

$$
J_{\lambda}^{1} E=\lambda^{*} J^{1} E=\left\{(q, Z) \in E \times J^{1} E|\lambda(q)=T \pi|_{J^{1} E}(Z)\right\},
$$

but the fibration we want to focus on is not one of the projections which define $J_{\lambda}^{1} E$, but rather the map $\pi_{1}=(\tau \circ \pi)_{1}^{0} \circ \lambda^{1}$. As such, we are looking at an affine bundle, modelled on the vector bundle $\bar{\pi}_{1}: V_{\rho} E \rightarrow E$, with total space

$$
V_{\rho} E=\left\{(\boldsymbol{v}, \boldsymbol{V}) \in V \times V E|\rho(\boldsymbol{v})=T \pi|_{V E}(\boldsymbol{V})\right\} .
$$

The affine bundles involved, and their underlying vector bundles are illustrated below. 

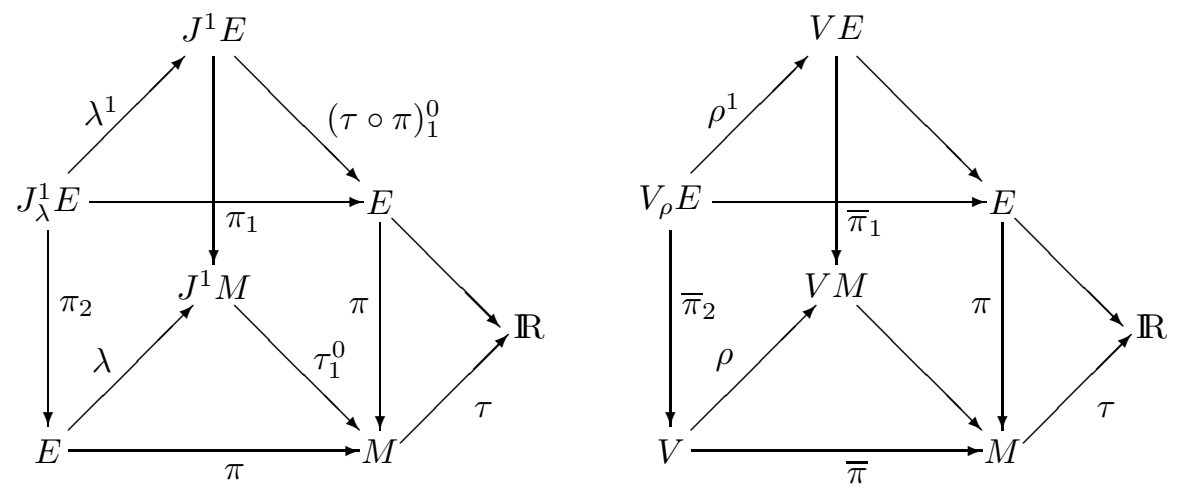

A section $Z$ of $\pi_{1}$ is completely determined once we know the maps $\pi_{2} \circ Z: E \rightarrow E$ and $\lambda^{1} \circ Z: E \rightarrow J^{1} E$. Likewise, vector sections $\boldsymbol{Z}$ of $\bar{\pi}_{1}$ are determined by $\bar{\pi}_{2} \circ \boldsymbol{Z}$ and $\rho^{1} \circ \boldsymbol{Z}$. For example, let $e \in E$ be a point with coordinates $\left(t, x^{i}, y^{\alpha}\right)$, so that $\left(t, x^{i}\right)$ are the coordinates of $\pi(e) \in M$ and $e$ has the representation $e=e_{0}+y^{\alpha} \boldsymbol{e}_{\alpha}$. If then $Z$ is a section of $\pi_{1}$, we will have:

$$
\begin{array}{ll}
\pi_{2} \circ Z: & (t, x, y) \longmapsto\left(t, x, z^{\alpha}(t, x, y)\right), \\
\lambda^{1} \circ Z: & \left.(t, x, y) \longmapsto\left(\frac{\partial}{\partial t}+\left(\lambda^{i}+\rho_{\alpha}^{i} z^{\alpha}\right) \frac{\partial}{\partial x^{i}}+Z^{\alpha} \frac{\partial}{\partial y^{\alpha}}\right)\right|_{e},
\end{array}
$$

and determining $Z$ in coordinates of course amounts to assigning the functions $\left(z^{\alpha}, Z^{\alpha}\right)$ on $E$.

It is worthwhile looking at the representation of such a $Z$ with respect to suitably selected local sections of $\pi_{1}$ and $\bar{\pi}_{1}$, which will exhibit the affine structure of $\pi_{1}$ and are adapted to the basis which was selected to coordinatize $E$. To this end, we introduce two sets of local sections $\mathcal{X}_{\alpha}$ and $\mathcal{V}_{\alpha}$ of $\bar{\pi}_{1}$ which will span $\operatorname{Sec}\left(\bar{\pi}_{1}\right)$, and select a zero section $\mathcal{E}_{0}$ as follows. The $\mathcal{V}_{\alpha}$ span 'vertical sections' and are determined by: $\bar{\pi}_{2} \circ \mathcal{V}_{\alpha}=\mathbf{0}$, while for $e \in E_{m}$ we let $\rho^{1} \circ \mathcal{V}_{\alpha}(e)$ be the tangent vector to the curve $s \mapsto e+s \boldsymbol{e}_{\alpha}(m)$ in $E_{m}$. Verticality is an intrinsic property whereas, as usual, there is no intrinsic notion of horizontality. The determination of the $\mathcal{X}_{\alpha}$ and $\mathcal{E}_{0}$ will therefore rely on pure coordinate arguments. For the projection onto $V$ we put $\bar{\pi}_{2} \circ \mathcal{X}_{\alpha}=\boldsymbol{e}_{\alpha} \circ \pi$ and then, fixing $\rho^{1} \circ \mathcal{X}_{\alpha}$ (as a vector field on $E)$ further requires making a prescription for the vertical components, which we simply take to be zero. Similarly, for the choice of a zero section, we could take any vector field on $E$ which projects under $T \pi$ onto $\lambda\left(e_{0}\right) \in \mathcal{X}(M)$ (and as such defines also a section of $\pi_{1}$ ), but we will fix it also by taking the vertical components to be zero. Thus we have:

$$
\boldsymbol{\mathcal { X }}_{\alpha}(e)=\left(\boldsymbol{e}_{\alpha}(\pi(e)),\left.\rho_{\alpha}^{i}(t, x) \frac{\partial}{\partial x^{i}}\right|_{e}\right) \quad \mathcal{V}_{\alpha}(e)=\left(\mathbf{0}(\pi(e)),\left.\frac{\partial}{\partial y^{\alpha}}\right|_{e}\right)
$$

and

$$
\mathcal{E}_{0}(e)=\left(e_{0}(\pi(e)),\left.\left(\frac{\partial}{\partial t}+\lambda^{i}(t, x) \frac{\partial}{\partial x^{i}}\right)\right|_{e}\right)
$$


The general section $Z$ of $\pi_{1}$ then has the local representation:

$$
Z=\mathcal{E}_{0}+z^{\alpha}(t, x, y) \mathcal{X}_{\alpha}+Z^{\alpha}(t, x, y) \mathcal{V}_{\alpha}
$$

Note that pseudo-SoDEs, as discussed in the previous section, are precisely those sections $\Gamma$ of $\pi_{1}$, for which $z^{\alpha}(t, x, y)=y^{\alpha}$.

Let now $E$ be equipped with an affine Lie algebroid structure. To be in line with the notations we used in Definition 1, we will from now on also write $\lambda^{1}(Z)$ instead of $\lambda^{1} \circ Z$, and likewise for the $\pi_{2}$-projection and the corresponding projections of vector sections.

We wish to establish that there is an induced Lie algebroid structure on the affine bundle $\pi_{1}$. To this end, following the scheme of Definition 1 , we have to identify a bracket on $\bar{\pi}_{1}$ and an action of affine sections on vector sections, such that all the necessary requirements are met. The idea is to define such brackets by requiring roughly that its two projections are determined by the known brackets of the projected sections. But there are some technical complications which we will address now.

For $\boldsymbol{Z}_{1}, \boldsymbol{Z}_{2} \in \operatorname{Sec}\left(\bar{\pi}_{1}\right)$, a preliminary observation is that the Lie bracket of their image under $\rho^{1}$ (which gives rise to vector fields on $E$ ), belongs to the image of $\rho^{1}$. A coordinate calculation can confirm this. Putting

$$
\rho^{1}\left(\boldsymbol{Z}_{i}\right)=z_{i}^{\alpha} \rho_{\alpha}^{j} \frac{\partial}{\partial x^{j}}+Z_{i}^{\alpha} \frac{\partial}{\partial y^{\alpha}}
$$

we have

$$
\begin{aligned}
{\left[\rho^{1}\left(\boldsymbol{Z}_{1}\right), \rho^{1}\left(\boldsymbol{Z}_{2}\right)\right]=} & \left(\rho^{1}\left(\boldsymbol{Z}_{1}\right)\left(z_{2}^{\alpha}\right)-\rho^{1}\left(\boldsymbol{Z}_{2}\right)\left(z_{1}^{\alpha}\right)\right) \rho_{\alpha}^{j} \frac{\partial}{\partial x^{j}} \\
& +\left(z_{2}^{\alpha} \rho^{1}\left(\boldsymbol{Z}_{1}\right)\left(\rho_{\alpha}^{j}\right)-z_{1}^{\alpha} \rho^{1}\left(\boldsymbol{Z}_{2}\right)\left(\rho_{\alpha}^{j}\right)\right) \frac{\partial}{\partial x^{j}}+\cdots \frac{\partial}{\partial y^{\alpha}} .
\end{aligned}
$$

The first term on the right manifestly belongs to the image of $\rho^{1}$, whereas the last term is irrelevant for that purpose. The middle term can be rewritten as

$$
z_{2}^{\alpha} z_{1}^{\beta}\left(\rho_{\beta}^{j} \frac{\partial \rho_{\alpha}^{i}}{\partial x^{j}}-\rho_{\alpha}^{j} \frac{\partial \rho_{\beta}^{i}}{\partial x^{j}}\right) \frac{\partial}{\partial x^{i}},
$$

which is seen to belong to the image of $\rho^{1}$ in view of the property (23). It is therefore natural to impose right away that the bracket $[\cdot, \cdot]^{1}$ under construction, which of course is required to be skew-symmetric and $\mathbb{R}$-bilinear, should satisfy

$$
\rho^{1}\left(\left[\boldsymbol{Z}_{1}, \boldsymbol{Z}_{2}\right]^{1}\right)=\left[\rho^{1}\left(\boldsymbol{Z}_{1}\right), \rho^{1}\left(\boldsymbol{Z}_{2}\right)\right]
$$

This will have for consequence that for $F_{i} \in C^{\infty}(E)$,

$$
\begin{aligned}
& \rho^{1}\left(\left[F_{1} \boldsymbol{Z}_{1}, F_{2} \boldsymbol{Z}_{2}\right]^{1}\right)= \\
& \quad F_{1} F_{2}\left[\rho^{1}\left(\boldsymbol{Z}_{1}\right), \rho^{1}\left(\boldsymbol{Z}_{2}\right)\right]+F_{1} \rho^{1}\left(\boldsymbol{Z}_{1}\right)\left(F_{2}\right) \rho^{1}\left(\boldsymbol{Z}_{2}\right)-F_{2} \rho^{1}\left(\boldsymbol{Z}_{2}\right)\left(F_{1}\right) \rho^{1}\left(\boldsymbol{Z}_{1}\right) .
\end{aligned}
$$


It remains then to make sure that the projection under $\bar{\pi}_{2}$ can be specified in a compatible way. The above coordinate calculation to some extent illustrates how one should proceed. If we apply $T \pi$ to the preceding equality, we get (pointwise)

$$
\begin{aligned}
& T \pi\left(\rho^{1}\left(\left[F_{1} \boldsymbol{Z}_{1}, F_{2} \boldsymbol{Z}_{2}\right]^{1}\right)\right)=\left(F_{1} F_{2}\right) T \pi\left(\left[\rho^{1}\left(\boldsymbol{Z}_{1}\right), \rho^{1}\left(\boldsymbol{Z}_{2}\right)\right]\right) \\
& \quad+F_{1} \rho^{1}\left(\boldsymbol{Z}_{1}\right)\left(F_{2}\right) \rho\left(\bar{\pi}_{2}\left(\boldsymbol{Z}_{2}\right)\right)-F_{2} \rho^{1}\left(\boldsymbol{Z}_{2}\right)\left(F_{1}\right) \rho\left(\bar{\pi}_{2}\left(\boldsymbol{Z}_{1}\right)\right) .
\end{aligned}
$$

In general, the $\rho\left(\bar{\pi}_{2}\left(\boldsymbol{Z}_{i}\right)\right)$ are vector fields along $\pi$ for which there is no standard Lie bracket available. If the $\boldsymbol{Z}_{i}$ are projectable, however, meaning that there exist $\boldsymbol{\zeta}_{i} \in \operatorname{Sec}(\bar{\pi})$ such that $\bar{\pi}_{2} \circ \boldsymbol{Z}_{i}=\boldsymbol{\zeta}_{i} \circ \pi$, the vector fields $\rho^{1}\left(\boldsymbol{Z}_{i}\right)$ on $E$ are $\pi$-related to the vector fields $\rho\left(\boldsymbol{\zeta}_{i}\right)$ on $M$. Hence, the corresponding brackets are also $\pi$-related, meaning that for projectable $\boldsymbol{Z}_{i}$, we can put

$$
\bar{\pi}_{2}\left(\left[\boldsymbol{Z}_{1}, \boldsymbol{Z}_{2}\right]^{1}\right)=\left[\bar{\pi}_{2}\left(\boldsymbol{Z}_{1}\right), \bar{\pi}_{2}\left(\boldsymbol{Z}_{2}\right)\right]
$$

and then the property (13) (which in coordinates gives (23)) ensures that

$$
T \pi\left(\rho^{1}\left(\left[\boldsymbol{Z}_{1}, \boldsymbol{Z}_{2}\right]^{1}\right)\right)=\rho \circ \bar{\pi}_{2}\left(\left[\boldsymbol{Z}_{1}, \boldsymbol{Z}_{2}\right]^{1}\right)
$$

as it should. The expression for $T \pi\left(\rho^{1}\left(\left[F_{1} \boldsymbol{Z}_{1}, F_{2} \boldsymbol{Z}_{2}\right]^{1}\right)\right)$ further shows that the $\bar{\pi}_{2}$ and $\rho^{1}$ projections of the bracket under construction will still match up if for projectable $\boldsymbol{Z}_{i}$ and for any $F_{i} \in C^{\infty}(E)$, we define

$$
\begin{aligned}
& \bar{\pi}_{2}\left(\left[F_{1} \boldsymbol{Z}_{1}, F_{2} \boldsymbol{Z}_{2}\right]^{1}\right)=F_{1} F_{2}\left[\bar{\pi}_{2}\left(\boldsymbol{Z}_{1}\right), \bar{\pi}_{2}\left(\boldsymbol{Z}_{2}\right)\right] \\
& \quad+F_{1} \rho^{1}\left(\boldsymbol{Z}_{1}\right)\left(F_{2}\right) \bar{\pi}_{2}\left(\boldsymbol{Z}_{2}\right)-F_{2} \rho^{1}\left(\boldsymbol{Z}_{2}\right)\left(F_{1}\right) \bar{\pi}_{2}\left(\boldsymbol{Z}_{1}\right) .
\end{aligned}
$$

It then follows that

$$
\left[F_{1} \boldsymbol{Z}_{1}, F_{2} \boldsymbol{Z}_{2}\right]^{1}=F_{1} F_{2}\left[\boldsymbol{Z}_{1}, \boldsymbol{Z}_{2}\right]^{1}+F_{1} \rho^{1}\left(\boldsymbol{Z}_{1}\right)\left(F_{2}\right) \boldsymbol{Z}_{2}-F_{2} \rho^{1}\left(\boldsymbol{Z}_{2}\right)\left(F_{1}\right) \boldsymbol{Z}_{1},
$$

since both sides have the same $\bar{\pi}_{2}$ and $\rho^{1}$ projections.

The final point to observe now is that sections of $\bar{\pi}_{1}$ (locally) are finitely generated, over the ring $C^{\infty}(E)$, by projectable sections. Hence, the defining relations $(\sqrt{76})$ and $(\sqrt{78})$ are sufficient to define the bracket $[\cdot, \cdot]^{1}$ on vector sections. The property (79) will hold by extension for all vector sections and the bracket will satisfy the Jacobi identity as a result of the Jacobi identity of the Lie algebroid bracket we start from and the same identity for vector fields on $E$.

To define the action of $Z \in \operatorname{Sec}\left(\pi_{1}\right)$ on $\boldsymbol{V} \in \operatorname{Sec}\left(\bar{\pi}_{1}\right)$, we proceed in exactly the same manner. First, one easily verifies that the Lie bracket of $\lambda^{1}(Z)$ and $\rho^{1}(\boldsymbol{V})$ belongs to the image of $\rho^{1}$, this time in view of the properties (22) and (23). Hence, it makes sense to put

$$
\rho^{1}\left([Z, \boldsymbol{V}]^{1}\right)=\left[\lambda^{1}(Z), \rho^{1}(\boldsymbol{V})\right]
$$

and we of course require the bracket $[\cdot, \cdot]^{1}$ to have linearity properties of the kind of (7)). For projectable sections, we can put

$$
\bar{\pi}_{2}\left([Z, \boldsymbol{V}]^{1}\right)=\left[\pi_{2}(Z), \bar{\pi}_{2}(\boldsymbol{V})\right]
$$


and be assured of consistency with the projection (80). Next, still for projectable $Z$ and $\boldsymbol{V}$ and for any $F \in C^{\infty}(E)$, we define

$$
\bar{\pi}_{2}\left([Z, F \boldsymbol{V}]^{1}\right)=F\left[\pi_{2}(Z), \bar{\pi}_{2}(\boldsymbol{V})\right]+\lambda^{1}(Z)(F) \bar{\pi}_{2}(\boldsymbol{V})
$$

Sections of $\pi_{1}$ can be written as a projectable zero section, plus a linear combination of projectable vector sections with coefficients in $C^{\infty}(E)$. In combination with the earlier arguments for vector sections, we are again led to the conclusion that the requirements (80) and (82) are sufficient to define $[Z, \boldsymbol{V}]^{1}$ for arbitrary $Z \in \operatorname{Sec}\left(\pi_{1}\right)$ and $\boldsymbol{V} \in \operatorname{Sec}\left(\bar{\pi}_{1}\right)$ and that we will have the property

$$
[Z, F \boldsymbol{V}]^{1}=F[Z, \boldsymbol{V}]^{1}+\lambda^{1}(Z)(F) \boldsymbol{V}
$$

The final requirement of type (8) then also easily follows, which concludes the construction of the prolonged affine Lie algebroid.

For computational purposes, it remains to list the brackets of the local sections which are used in the general representation of a section of $\pi_{1}$ as in (75). We have

$$
\begin{array}{ll}
{\left[\mathcal{E}_{0}, \mathcal{X}_{\alpha}\right]^{1}=C_{\alpha}^{\beta} \mathcal{X}_{\beta},} & {\left[\mathcal{E}_{0}, \mathcal{V}_{\alpha}\right]^{1}=\mathbf{0},} \\
{\left[\mathcal{X}_{\alpha}, \mathcal{X}_{\beta}\right]^{1}=C_{\alpha \beta}^{\gamma} \mathcal{X}_{\gamma},} & {\left[\mathcal{X}_{\alpha}, \mathcal{V}_{\beta}\right]^{1}=\mathbf{0}, \quad\left[\mathcal{V}_{\alpha}, \mathcal{V}_{\beta}\right]^{1}=\mathbf{0}}
\end{array}
$$

It is perhaps worthwhile to repeat hereby that the two projections have to be looked at to verify these statements, although of course they are bound to match up if our new bracket has been defined consistently. Thus we have, for example:

$$
\begin{aligned}
\bar{\pi}_{2}\left(\left[\mathcal{E}_{0}, \boldsymbol{\mathcal { X }}_{\alpha}\right]^{1}\right) & =\left[\pi_{2}\left(\mathcal{E}_{0}\right), \bar{\pi}_{2}\left(\boldsymbol{\mathcal { X }}_{\alpha}\right)\right]=\left[e_{0}, \boldsymbol{e}_{\alpha}\right]=C_{\alpha}^{\beta} \boldsymbol{e}_{\beta}, \\
\rho^{1}\left(\left[\mathcal{E}_{0}, \boldsymbol{\mathcal { X }}_{\alpha}\right]^{1}\right) & =\left[\lambda^{1}\left(\mathcal{E}_{0}\right), \rho^{1}\left(\boldsymbol{\mathcal { X }}_{\alpha}\right)\right]=\left[\frac{\partial}{\partial t}+\lambda^{i} \frac{\partial}{\partial x^{i}}, \rho_{\alpha}^{j} \frac{\partial}{\partial x^{j}}\right]=C_{\alpha}^{\beta} \rho^{1}\left(\boldsymbol{\mathcal { X }}_{\beta}\right),
\end{aligned}
$$

where (22) has been used again in the last line.

\section{Discussion and outlook for future work}

The form of equations (4), which we claim to be the appropriate generalization of Lagrangian systems on Lie algebroids to a situation where explicit time-dependence is involved, has brought us to the introduction of the new concept of Lie algebroids on affine bundles which are fibred over $\mathbb{R}$. More precisely, the first guidance for developping this concept was provided by the conditions (5) and (6) which the various functions appearing in (4) have to satisfy. Ultimately, of course, we want to arrive at an intrinsic geometrical construction of such Lagrangian systems. There are many aspects to be explored yet, but we have sufficiently paved the way already to be able to predict what the outcome of subsequent studies will bring.

One of us has shown [10 that the prolongation of a Lie algebroid (in the standard situation of vector bundles) provides a platform where there exist analogues of the intrinsic 
structures living on a tangent bundle and these in turn give rise to an intrinsic definition of Lagrangian systems via Poincaré-Cartan type forms. This is the reason why we were keen to verify immediately that the same notion of prolongation exists in our affine set-up. There is little doubt now that we will find intrinsic objects on such a prolonged affine Lie algebroid, which are analogues of what is known to give rise to an intrinsic definition of time-dependent Lagrangian systems on the first jet bundle of a manifold fibred over $\mathbb{R}$. But there is more to it. Even when there is no Lagrangian for the dynamics under consideration and we are, in other words, talking about pseudo-SoDEs on a Lie algebroid, we expect to be able to develop a machinery of associated non-linear and linear connections, which again is analogous to the standard theory of connections associated to SoDEs on a tangent bundle or first jet bundle. In fact, a paper on these issues for the case of Lie algebroids on a vector bundle is in preparation.

One of the features we examined in this paper as a kind of test for the relevance and internal consistency of the generalized notion of Lie algebroids, was the existence of an associated coboundary operator $d$. But of course, there are still other interesting properties which standard Lie algebroids are known to exhibit. Let us briefly highlight another one here and show that it also survives our generalization, namely the existence of an associated Poisson structure. Specifically, we want to establish that there exists a canonically defined Poisson structure on the extended dual $E^{\dagger}$.

Sections of $\pi$ (respectively $\bar{\pi}$ ) can be identified with linear functions on $E^{\dagger}$ (respectively $\left.V^{*}\right)$. Explicitly, if $\zeta \in \operatorname{Sec}(\pi)$, we consider the function $\hat{\zeta} \in C^{\infty}\left(E^{\dagger}\right)$ defined by: for each $p \in E^{\dagger}, p \in E_{m}^{\dagger}$ say, $\hat{\zeta}(p)=p\left(\zeta_{m}\right)$. Likewise, if $\boldsymbol{\sigma} \in \operatorname{Sec}(\bar{\pi})$, we denote by $\hat{\boldsymbol{\sigma}} \in C^{\infty}\left(V^{*}\right)$ the function defined by $\hat{\boldsymbol{\sigma}}(\boldsymbol{p})=\boldsymbol{p}\left(\boldsymbol{\sigma}_{m}\right)$, where $\boldsymbol{p} \in V_{m}^{*}$. In coordinates, if $\zeta=e_{0}+\zeta^{\alpha} \boldsymbol{e}_{\alpha}$ and $p \in E^{\dagger}$ has coordinates $\left(t, x^{i}, p_{0}, p_{\alpha}\right)$, then $\hat{\zeta}(p)=p_{0}+p_{\alpha} \zeta^{\alpha}(t, x)$ and similarly for $\hat{\boldsymbol{\sigma}}$. Now, for any two sections $\zeta, \eta \in \operatorname{Sec}(\pi)$, we define the function $\{\hat{\zeta}, \hat{\eta}\}$ on $E^{\dagger}$ by

$$
\{\hat{\zeta}, \hat{\eta}\}(p)=\widehat{[\zeta, \eta]}(\boldsymbol{p}),
$$

whereby we recall that $[\zeta, \eta]$ is a section of $\bar{\pi}$ and for $p \in E_{m}^{\dagger}, \boldsymbol{p}$ is the associated element of $V_{m}^{*}$. If further $f, g$ are functions on $M$ and we make no notational distinction for their pullback to $E^{\dagger}$, internal consistency of (84) for the action of $\operatorname{Sec}(\bar{\pi})$ on $\operatorname{Sec}(\pi)$ requires that we further put

$$
\{\hat{\zeta}, f\}=-\{f, \hat{\zeta}\}=\lambda(\zeta)(f), \quad\{f, g\}=0
$$

The construction then uniquely extends to a skew-symmetric, $\mathbb{R}$-bilinear bracket operation on $E^{\dagger}$ with the required derivation property. This bracket satisfies the Jacobi identity as a result of the Lie algebroid Jacobi identity (16).

The brackets for the coordinate functions on $E^{\dagger}$ are found to be:

$$
\begin{array}{lll}
\{t, t\}=0 & \left\{t, x^{i}\right\}=0 & \left\{x^{i}, x^{j}\right\}=0 \\
\left\{p_{0}, t\right\}=1 & \left\{p_{0}, x^{i}\right\}=\lambda^{i} & \left\{p_{0}, p_{\beta}\right\}=C_{\beta}^{\gamma} p_{\gamma} \\
\left\{p_{\alpha}, t\right\}=0 & \left\{p_{\alpha}, x^{i}\right\}=\rho_{\alpha}^{i} & \left\{p_{\alpha}, p_{\beta}\right\}=C_{\alpha \beta}^{\gamma} p_{\gamma}
\end{array}
$$


There is an interesting observation to be made here. Recall that $E^{\dagger}$ is actually a vector bundle and note now that the bracket we have constructed preserves the subset of functions on $E^{\dagger}$ which are linear in the fibre coordinates. As a result, we know that there is an induced Lie algebroid structure on the bundle $\left(E^{\dagger}\right)^{*} \rightarrow M$. There are many new insights to be gained from approaching the subject of a Lie algebroid structure on the affine bundle $E \rightarrow M$ from this angle; that is to say, by regarding $E \rightarrow M$ as an affine subbundle of $\left(E^{\dagger}\right)^{*} \rightarrow M$ and taking an appropriate Lie algebroid structure on $\left(E^{\dagger}\right)^{*} \rightarrow M$ as the starting point. Also this will be the subject of a forthcoming paper.

\section{Appendix: Technical proofs}

The start for proving Proposition 3 is the defining relation (52) of the exterior derivative, in which we make use of the decomposition (26) in the first term and (51) in the second. We first obtain,

$$
\begin{aligned}
d \omega & \left(\zeta_{1}, \ldots, \zeta_{k+1}\right) \\
& =\sum_{i=1}^{k+1}(-1)^{i-1}\left(\lambda\left(\zeta_{0}\right)+\rho\left(\boldsymbol{\zeta}_{i}\right)\right)\left(\sum_{j=1}^{i-1}(-1)^{j-1} \omega_{0}\left(\zeta_{0}, \boldsymbol{\zeta}_{1}, \ldots, \hat{\boldsymbol{\zeta}}_{j}, \ldots, \hat{\boldsymbol{\zeta}}_{i}, \ldots, \boldsymbol{\zeta}_{k+1}\right)\right. \\
& \left.+\sum_{j=i+1}^{k+1}(-1)^{j} \omega_{0}\left(\zeta_{0}, \boldsymbol{\zeta}_{1}, \ldots, \hat{\boldsymbol{\zeta}}_{i}, \ldots, \hat{\boldsymbol{\zeta}}_{j}, \ldots, \boldsymbol{\zeta}_{k+1}\right)+\boldsymbol{\omega}\left(\zeta_{1}, \ldots, \hat{\boldsymbol{\zeta}}_{i}, \ldots, \boldsymbol{\zeta}_{k+1}\right)\right) \\
& +\sum_{1 \leq i<j \leq k+1}(-1)^{i+j}\left(\boldsymbol{\omega}\left(\left[\zeta_{i}, \zeta_{j}\right], \boldsymbol{\zeta}_{1}, \ldots, \hat{\boldsymbol{\zeta}}_{i}, \ldots, \hat{\boldsymbol{\zeta}}_{j}, \ldots, \boldsymbol{\zeta}_{k+1}\right)\right. \\
& +\sum_{l=1}^{i-1}(-1)^{l} \omega_{0}\left(\zeta_{0},\left[\zeta_{i}, \zeta_{j}\right], \boldsymbol{\zeta}_{1}, \ldots, \hat{\boldsymbol{\zeta}}_{l}, \ldots, \hat{\boldsymbol{\zeta}}_{i}, \ldots, \hat{\boldsymbol{\zeta}}_{j}, \ldots, \boldsymbol{\zeta}_{k+1}\right) \\
& +\sum_{l=i+1}^{j-1}(-1)^{l-1} \omega_{0}\left(\zeta_{0},\left[\zeta_{i}, \zeta_{j}\right], \boldsymbol{\zeta}_{1}, \ldots, \hat{\boldsymbol{\zeta}}_{i}, \ldots, \hat{\boldsymbol{\zeta}}_{l}, \ldots, \hat{\boldsymbol{\zeta}}_{j}, \ldots, \boldsymbol{\zeta}_{k+1}\right) \\
& \left.+\sum_{l=j+1}^{k+1}(-1)^{l} \omega_{0}\left(\zeta_{0},\left[\zeta_{i}, \zeta_{j}\right], \boldsymbol{\zeta}_{1}, \ldots, \hat{\boldsymbol{\zeta}}_{i}, \ldots, \hat{\boldsymbol{\zeta}}_{j}, \ldots, \hat{\boldsymbol{\zeta}}_{l}, \ldots, \boldsymbol{\zeta}_{k+1}\right)\right),
\end{aligned}
$$

and now perform a number of manipulations on multiple sums. Interchanging the order of summation in the first line, we have $\sum_{i=1}^{k+1} \sum_{j=1}^{i-1}=\sum_{j=1}^{k} \sum_{i=j+1}^{k+1}$. Interchanging subsequently the names of the indices $i$ and $j$, the term involving $\lambda\left(\zeta_{0}\right)$ of the first line cancels the similar one in the second line. The last three lines involve triple sums, which can be rearranged as follows. The first triple sum, with suitable interchanges of the order of summation, becomes:

$$
\sum_{i=1}^{k} \sum_{j=i+1}^{k+1} \sum_{l=1}^{i-1}=\sum_{i=1}^{k} \sum_{l=1}^{i-1} \sum_{j=i+1}^{k+1}=\sum_{l=1}^{k-1} \sum_{i=l+1}^{k} \sum_{j=i+1}^{k+1}=\sum_{1 \leq l<i<j \leq k+1} .
$$


For the second one, we have

$$
\sum_{i=1}^{k} \sum_{j=i+1}^{k+1} \sum_{l=i+1}^{j-1}=\sum_{i=1}^{k} \sum_{l=i+1}^{k} \sum_{j=l+1}^{k+1}=\sum_{1 \leq i<l<j \leq k+1}
$$

The last one can directly be written as $\sum_{1 \leq i<j<l \leq k+1}$. Changing names of indices to make all triple sums look alike, we thus far arrive at the result:

$$
\begin{aligned}
& d \omega\left(\zeta_{1}, \ldots, \zeta_{k+1}\right) \\
& \quad=\sum_{1 \leq i<j \leq k+1}(-1)^{i+j} \rho\left(\boldsymbol{\zeta}_{j}-\boldsymbol{\zeta}_{i}\right)\left(\omega_{0}\left(\zeta_{0}, \boldsymbol{\zeta}_{1}, \ldots, \hat{\boldsymbol{\zeta}}_{i}, \ldots, \hat{\boldsymbol{\zeta}}_{j}, \ldots, \boldsymbol{\zeta}_{k+1}\right)\right) \\
& \quad+\sum_{i=1}^{k+1}(-1)^{i-1}\left(\lambda\left(\zeta_{0}\right)+\rho\left(\boldsymbol{\zeta}_{i}\right)\right)\left(\boldsymbol{\omega}\left(\boldsymbol{\zeta}_{1}, \ldots, \hat{\boldsymbol{\zeta}}_{i}, \ldots, \boldsymbol{\zeta}_{k+1}\right)\right) \\
& \quad+\sum_{1 \leq i<j \leq k+1}(-1)^{i+j} \boldsymbol{\omega}\left(\left[\zeta_{0}, \boldsymbol{\zeta}_{j}\right]-\left[\zeta_{0}, \boldsymbol{\zeta}_{i}\right]+\left[\boldsymbol{\zeta}_{i}, \boldsymbol{\zeta}_{j}\right], \boldsymbol{\zeta}_{1}, \ldots, \hat{\boldsymbol{\zeta}}_{i}, \ldots, \hat{\boldsymbol{\zeta}}_{j}, \ldots, \boldsymbol{\zeta}_{k+1}\right) \\
& \quad+\sum_{1 \leq i<j<l \leq k+1}(-1)^{i+j+l} \omega_{0}\left(\zeta_{0},\left[\zeta_{i}, \zeta_{j}\right]+\left[\zeta_{j}, \zeta_{l}\right]+\left[\zeta_{l}, \zeta_{i}\right], \boldsymbol{\zeta}_{1}, ., \hat{\boldsymbol{\zeta}}_{i}, ., \hat{\boldsymbol{\zeta}}_{j}, ., \hat{\boldsymbol{\zeta}}_{l}, ., \boldsymbol{\zeta}_{k+1}\right) .
\end{aligned}
$$

It is clear now that the terms which do not involve $\zeta_{0}$ combine exactly to $d \boldsymbol{\omega}\left(\boldsymbol{\zeta}_{1}, \ldots, \boldsymbol{\zeta}_{k+1}\right)$. What remains is

$$
\begin{aligned}
& \sum_{i=1}^{k+1}(-1)^{i-1} \lambda\left(\zeta_{0}\right)\left(\boldsymbol{\omega}\left(\boldsymbol{\zeta}_{1}, \ldots, \hat{\boldsymbol{\zeta}}_{i}, \ldots, \boldsymbol{\zeta}_{k+1}\right)\right) \\
& +\sum_{1 \leq i<j \leq k+1}(-1)^{i+j} \rho\left(\boldsymbol{\zeta}_{j}-\boldsymbol{\zeta}_{i}\right)\left(\omega_{0}\left(\zeta_{0}, \boldsymbol{\zeta}_{1}, \ldots, \hat{\boldsymbol{\zeta}}_{i}, \ldots, \hat{\boldsymbol{\zeta}}_{j}, \ldots, \boldsymbol{\zeta}_{k+1}\right)\right) \\
& +\sum_{1 \leq i<j \leq k+1}(-1)^{i+j} \boldsymbol{\omega}\left(\left[\zeta_{0}, \boldsymbol{\zeta}_{j}\right]-\left[\zeta_{0}, \boldsymbol{\zeta}_{i}\right], \boldsymbol{\zeta}_{1}, \ldots, \hat{\boldsymbol{\zeta}}_{i}, \ldots, \hat{\boldsymbol{\zeta}}_{j}, \ldots, \boldsymbol{\zeta}_{k+1}\right) \\
& +\sum_{1 \leq i<j<l \leq k+1}(-1)^{i+j+l} \omega_{0}\left(\zeta_{0},\left[\zeta_{i}, \zeta_{j}\right]+\left[\zeta_{j}, \zeta_{l}\right]+\left[\zeta_{l}, \zeta_{i}\right], \boldsymbol{\zeta}_{1}, ., \hat{\boldsymbol{\zeta}}_{i}, ., \hat{\boldsymbol{\zeta}}_{j}, ., \hat{\boldsymbol{\zeta}}_{l}, ., \boldsymbol{\zeta}_{k+1}\right),
\end{aligned}
$$

and should be compared to $\sum_{i=1}^{k+1}(-1)^{i-1} d \omega_{0}\left(\zeta_{0}, \boldsymbol{\zeta}_{1}, \ldots, \hat{\boldsymbol{\zeta}}_{i}, \ldots, \boldsymbol{\zeta}_{k+1}\right)$, with $d \omega_{0}$ as defined in (53). It is obvious that the first three lines in the computation of $(d \omega)^{0}$ are exactly the ones we have in the above expression. The last term in (53) gives rise to triple sums of the form $\sum_{l=1}^{k+1}(-1)^{l-1} \sum_{i<j<l}(-1)^{i+j-1} \omega_{0}\left(\zeta_{0},\left[\boldsymbol{\zeta}_{i}, \boldsymbol{\zeta}_{j}\right], \boldsymbol{\zeta}_{1}, ., \boldsymbol{\zeta}_{i}, ., \boldsymbol{\zeta}_{j}, ., \boldsymbol{\zeta}_{l}, ., \boldsymbol{\zeta}_{k+1}\right)$ (there is a similar term with $\sum_{i<l<j}$ and one with $\sum_{l<i<j}$ ). With suitable interchanges of summations, similar to what was explicitly explained before, these three terms combine to:

$$
\sum_{1 \leq i<j<l \leq k+1}(-1)^{i+j+l} \omega_{0}\left(\zeta_{0},\left[\boldsymbol{\zeta}_{i}, \boldsymbol{\zeta}_{j}\right]+\left[\boldsymbol{\zeta}_{j}, \boldsymbol{\zeta}_{l}\right]+\left[\boldsymbol{\zeta}_{l}, \boldsymbol{\zeta}_{i}\right], \boldsymbol{\zeta}_{1}, ., \hat{\boldsymbol{\zeta}}_{i}, ., \hat{\boldsymbol{\zeta}}_{j}, ., \hat{\boldsymbol{\zeta}}_{l}, ., \boldsymbol{\zeta}_{k+1}\right) .
$$

The proof now becomes complete if we observe that:

$$
\left[\zeta_{i}, \zeta_{j}\right]+\left[\zeta_{j}, \zeta_{l}\right]+\left[\zeta_{l}, \zeta_{i}\right]=\left[\boldsymbol{\zeta}_{i}, \boldsymbol{\zeta}_{j}\right]+\left[\boldsymbol{\zeta}_{j}, \boldsymbol{\zeta}_{l}\right]+\left[\boldsymbol{\zeta}_{l}, \boldsymbol{\zeta}_{i}\right]
$$


We next turn to the proof of Lemma 2.

If $\omega$ is a $k$-form, then $d^{2} \omega$ is a $(k+2)$-form with

$$
\begin{aligned}
d^{2} \omega\left(\zeta_{1}, \ldots, \zeta_{k+2}\right)= & \sum_{i=1}^{k+2}(-1)^{i-1} \lambda\left(\zeta_{i}\right)\left(d \omega\left(\zeta_{1}, \ldots, \hat{\zeta}_{i}, \ldots, \zeta_{k+2}\right)\right) \\
& +\sum_{1 \leq i<j \leq k+2}(-1)^{i+j} d \omega\left(\left[\zeta_{i}, \zeta_{j}\right], \zeta_{1}, \ldots, \hat{\zeta}_{i}, \ldots, \hat{\zeta}_{j}, \ldots, \zeta_{k+2}\right)
\end{aligned}
$$

If we plug in the definition of $d \omega$, the first term on the right will further decompose into two parts, one involving double and the other involving triple sums. Based on our experience with such combinatorics in the preceding proof, we can right away conclude that the first line of the right-hand side of (86) equals:

$$
\begin{aligned}
& \sum_{1 \leq i<j \leq k+2}(-1)^{i+j}\left(\lambda\left(\zeta_{j}\right) \lambda\left(\zeta_{i}\right)-\lambda\left(\zeta_{i}\right) \lambda\left(\zeta_{j}\right)\right)\left(\omega\left(\zeta_{1}, \ldots, \hat{\zeta}_{i}, \ldots, \hat{\zeta}_{j}, \ldots, \zeta_{k+2}\right)\right) \\
& +\sum_{1 \leq i<j<l \leq k+2}(-1)^{i+j+l-1} \sum_{i, j, l}\left\{\lambda\left(\zeta_{i}\right)\left(\omega\left(\left[\zeta_{j}, \zeta_{l}\right]\right\}, \zeta_{1}, ., \hat{\zeta}_{i}, ., \hat{\zeta}_{j}, ., \hat{\zeta}_{l}, ., \zeta_{k+2}\right)\right),
\end{aligned}
$$

where the smaller summation sign, as before, refers to a cyclic sum, the range of which is delimited by the curly brackets. For the second term on the right in (86), we have to remember that the first argument is a vector section. Using the defining relation (50), applied to $d \omega$, we obtain:

$$
\begin{aligned}
& d \omega\left(\boldsymbol{\sigma}, \zeta_{1}, \ldots, \zeta_{k}\right)=\rho(\boldsymbol{\sigma})\left(\omega\left(\zeta_{1}, \ldots, \zeta_{k}\right)\right) \\
& \quad+\sum_{i=1}^{k}(-1)^{i} \lambda\left(\zeta_{i}\right)\left(\omega\left(\boldsymbol{\sigma}, \zeta_{1}, \ldots, \hat{\zeta}_{i}, \ldots, \zeta_{k}\right)\right) \\
& \quad+\sum_{j=1}^{k}(-1)^{j} \omega\left(\left[\boldsymbol{\sigma}, \zeta_{j}\right], \zeta_{1}, \ldots, \hat{\zeta}_{j}, \ldots, \zeta_{k}\right) \\
& \quad+\sum_{1 \leq i<j \leq k}(-1)^{i+j} \omega\left(\left[\zeta_{i}, \zeta_{j}\right], \boldsymbol{\sigma}, \zeta_{1}, \ldots, \hat{\zeta}_{i}, \ldots, \hat{\zeta}_{j}, \ldots, \zeta_{k}\right) .
\end{aligned}
$$

The last line here has two vector arguments, but this is consistent with the application of definition 5 to a form of type $i_{\zeta} \omega$. We look at the effect of each of these four terms, when inserted in the second sum of (86). The first one simply gives:

$$
\sum_{1 \leq<i<j \leq k+2}(-1)^{i+j} \rho\left(\left[\zeta_{i}, \zeta_{j}\right]\right)\left(\omega\left(\zeta_{1}, \ldots, \hat{\zeta}_{i}, \ldots, \hat{\zeta}_{j}, \ldots, \zeta_{k+2}\right)\right) .
$$

The second one is easily seen to give rise to terms which cancel exactly the second sum in (87). The third term of (88) gives rise to expressions involving double brackets, which combine to:

$$
\sum_{1 \leq i<j<l \leq k+2}(-1)^{i+j+l} \omega\left(\sum_{i, j, l}\left[\left[\zeta_{i}, \zeta_{j}\right], \zeta_{l}\right], \zeta_{1}, ., \hat{\zeta}_{i}, ., \hat{\zeta}_{j}, ., \hat{\zeta}_{l}, ., \zeta_{k+2}\right)
$$


The fourth term of (88) finally creates terms which involve two double sums, and in each of the summands the first two arguments of $\omega$ are brackets. One has to look at all possible orderings, six in total, of the four different indices involved, but when the same procedure is applied to shuffle the order of summations suitably around and rename indices where appropriate, one easily finds that the six terms cancel each other two by two in view of the skew-symmetry of $\omega$. What we are left with in the end is the first term of (87), (89) and (90): they precisely combine to the statement in Lemma 2.

\section{References}

[1] J.F. Cariñena, Lie groupoids and algebroids in classical and quantum mechanics, In: Symmetries in quantum mechanics and quantum optics, University of Burgos (Spain) (1999), $67-81$.

[2] J.F. Cariñena and E. Martínez, Lie algebroid generalization of geometric mechanics, In: Poisson Geometry, Banach Center Publications (2001), to appear.

[3] J. Clemente-Gallardo, Applications of Lie algebroids in mechanics and control theory, In: Nonlinear control in the new millenium, F. Lamnabhi-Lagarrigur, W. Respondek and A. Isidori, eds., (Springer Verlag) (2000).

[4] M. Crampin, Jet bundle techniques in analytical mechanics, Quaderni del consiglio nazionale delle ricerche, gruppo nazionale di fisica matematica 47 (1995).

[5] A. Frölicher and A. Nijenhuis, Theory of vector-valued differential forms, Proc. Ned. Acad. Wetensch. Sér. A 59 (1956) 338-359.

[6] J. Grabowski and P. Urbanski, Tangent and cotangent lifts and graded Lie algebras associated with Lie algebroids, Ann. Global An. Geom. 15 (1997) 447-486.

[7] J. Grabowski and P. Urbanski, Lie algebroids and Poisson-Nijenhuis structures, Rep. Math. Phys. 40 (1997) 195-208.

[8] P. Libermann, Lie algebroids and Mechanics, Arch. Math. (Brno) 32 (1996) 147-162.

[9] K. Mackenzie, Lie groupoids and Lie algebroids in differential geometry, London Math. Soc. Lect. Note Series 124 (Cambridge Univ. Press) (1987).

[10] E. Martínez, Lagrangian Mechanics on Lie algebroids, Acta. Appl. Math. 67 (2001) 295-320.

[11] E. Martínez, J.F. Cariñena and W. Sarlet, Derivations of differential forms along the tangent bundle projection II, Diff. Geometry and its Applications 3 (1993) 1-29.

[12] W. Sarlet, T. Mestdag and E. Martínez, Lagrangian equations on affine Lie algebroids, In: Differential Geometry and its Applications, Proc. 8th Int. Conf. (Opava 2001), D. Krupka et al (Eds.), to appear.

[13] A. Weinstein, Lagrangian Mechanics and groupoids, In: Mechanics day (Waterloo, ON, 1992), Fields Institute Communications 7, American Mathematical Society (1996) 207231. 